ARTICLE

\title{
A role for actin flexibility in thin filament-mediated contractile regulation and myopathy
}

\author{
Meera C. Viswanathan ${ }^{1,6}$, William Schmidt ${ }^{1,6}$, Peter Franz (1) 2,6, Michael J. Rynkiewicz ${ }^{3}$, \\ Christopher S. Newhard ${ }^{4}$, Aditi Madan', William Lehman ${ }^{3}$, Douglas M. Swank ${ }^{4}$, Matthias Preller (i) ${ }^{2 凶} \&$ \\ Anthony Cammarato $1,5 \otimes$
}

Striated muscle contraction is regulated by the translocation of troponin-tropomyosin strands over the thin filament surface. Relaxation relies partly on highly-favorable, conformationdependent electrostatic contacts between actin and tropomyosin, which position tropomyosin such that it impedes actomyosin associations. Impaired relaxation and hypercontractile properties are hallmarks of various muscle disorders. The $\alpha$-cardiac actin M305L hypertrophic cardiomyopathy-causing mutation lies near residues that help confine tropomyosin to an inhibitory position along thin filaments. Here, we investigate M305 L actin in vivo, in vitro, and in silico to resolve emergent pathological properties and disease mechanisms. Our data suggest the mutation reduces actin flexibility and distorts the actin-tropomyosin electrostatic energy landscape that, in muscle, result in aberrant contractile inhibition and excessive force. Thus, actin flexibility may be required to establish and maintain interfacial contacts with tropomyosin as well as facilitate its movement over distinct actin surface features and is, therefore, likely necessary for proper regulation of contraction.

\footnotetext{
${ }^{1}$ Department of Medicine, Division of Cardiology, Johns Hopkins University, 720 Rutland Avenue, Baltimore, MD 21205, USA. ${ }^{2}$ Institute for Biophysical Chemistry, Hannover Medical School, Carl-Neuberg-Straße 1, 30625 Hannover, Germany. ${ }^{3}$ Department of Physiology and Biophysics, Boston University School of Medicine, 700 Albany Street St, Boston, MA 02118, USA. ${ }^{4}$ Department of Biological Sciences and Center for Biotechnology and Interdisciplinary Studies, Rensselaer Polytechnic Institute, 110 8th Street, Troy, NY 12180-3590, USA. ${ }^{5}$ Department of Physiology, Johns Hopkins University School of Medicine, 720 Rutland Avenue, Baltimore, MD 21205, USA. ${ }^{6}$ These authors contributed equally: Meera C. Viswanathan, William Schmidt, Peter Franz.

凶email: Preller.Matthias@mh-hannover.de; acammar3@jhmi.edu
} 
P roper regulation of myocardial contraction and relaxation is essential for the heart to effectively pump blood, and even the most subtle disruptions in either process can elicit disease. Hypertrophic cardiomyopathy (HCM) is a heterogeneous heart disorder, with a prevalence estimated at 1 in 200, that is characterized by asymmetric myocardial growth, thickening of the interventricular septum, hyperdynamic contractile properties during systole, and impaired relaxation during diastole ${ }^{1-3}$. More than 1500 mutations in at least 11 genes are associated with HCM, which frequently engender a gain-in-sarcomericfunction ${ }^{1,2,4-6}$. Since the initial identification of an $\alpha$-cardiac actin (ACTC) HCM-causing mutation in 1999, at least 12 additional ACTC variants have been reported in patients with HCM. The loci of the mutations are broadly distributed over the molecule with the M305L substitution located in subdomain 3 (SD3), close to the nucleotide-binding site (Supplementary Fig. 1A-C) ${ }^{7}$. Originally discovered in 2004, studies of M305L actin have focused predominantly on isolated protein measurements of mutationinduced changes in stability, polymerization properties, and nucleotide/phosphate release $\mathrm{e}^{8-11}$. Much of the data are inconsistent, and in some cases contradictory, which confounds our understanding of the pathological basis of disease. Therefore, extending these analyses, to determine the mutation's effects in situ and/or in vivo, may help resolve how M305L actin elicits HCM and, importantly, highlight universal properties of muscle biology. To date, such studies are lacking due to an inability to express M305L actin in cultured cardiomyocytes ${ }^{10}$ and the absence of animal models.

Cardiac muscle contraction is driven by cyclic interactions between myosin S1 heads of thick filaments and actin-containing thin filaments. It is regulated by troponin-tropomyosin (Tn-Tpm) complexes that, at rest, occlude myosin binding sites along filamentous actin (F-actin) and limit S1-thin filament binding ${ }^{12,13}$. Specifically, when sarcoplasmic $\mathrm{Ca}^{2+}$ is low, Tn binds to F-actin and Tpm and constrains Tpm to the B-state where it blocks and restricts myosin attachment. When elevated, $\mathrm{Ca}^{2+}$ binds to Tn, prompting the azimuthal movement of Tpm to the C-state that partially uncovers myosin binding sites. This facilitates weak-to-strong S1 cross-bridge binding and further displacement of Tpm to the M-state, which promotes additional S1 binding and contraction. During relaxation, following myosin detachment from F-actin, recent data suggest Tpm spontaneously moves back toward the energetically favorable, inhibitory B-state configuration $^{14}$. Thus, Tpm must seamlessly translocate backand-forth over the surface of F-actin, including any topological amino acid protrusions, to effectively regulate S1-thin filament cycling and, thereby, permit or prevent the production of force.

$\mathrm{Tpm}$ is an alpha-helical, coiled-coil dimer that binds seven adjacent actin monomers ${ }^{15-17}$. The dimers self-polymerize to produce a continuous strand that wraps around the F-actin helix. Locally, individual dimers associate weakly with the thin filament; yet, the full-length Tpm cable as a whole interacts strongly with F-actin ${ }^{18-21}$. This purported Gestalt-binding behavior of Tpm permits high affinity global binding that prevents detachment of the cable from F-actin, while low affinity local binding allows for the dynamic, azimuthal movements that are imperative for Tpm's regulatory role in striated muscle ${ }^{21,22}$. Gestalt-binding relies on high complementarity between the three-dimensional, preshaped contours of Tpm and F-actin ${ }^{19,21,23}$. The structural complementarity permits the formation of roughly 30 interfacial electrostatic contacts that were predicted to establish the aforementioned weak, local F-actin-Tpm interactions and a broad energy basin, which biases Tpm to a location that limits S1-Factin binding 24 . This energetically stable F-actin-Tpm conformation, known as the A-state, places Tpm in a location that is effectively the same as its B-state position on thin filaments replete with $\mathrm{Tn}$; however, when not pinned down by $\mathrm{Ca}^{2+}$-free Tn, Tpm has greater azimuthal freedom ${ }^{21}$. Several actin residues, including K326, K328, and E334, are located along a continuous stretch of amino acids of SD3 and associate extensively with Tpm to help establish the A-state ${ }^{24-28}$. Notably, in vitro and in vivo findings illustrate that these associations facilitate Tn-Tpmmediated steric blocking of S1 and, therefore, contribute to proper relaxation of cardiac and skeletal muscle $22,29-31$.

Here, we present a study that comprehensively assesses the consequences of $\mathrm{M} 305 \mathrm{~L}$ actin on striated muscle at the tissue, cellular, and molecular levels. Given the limited and often paradoxical findings regarding the intrinsic properties of M305L actin, we hypothesize that the lesion's effects would be maximally manifested in higher order contractile systems replete with regulatory components and, ultimately, the mutation disrupts Tpm positioning and actomyosin inhibition. We show that in the Drosophila melanogaster heart, M305L mutant actin incorporates uniformly down cardiac thin filaments, increases periods of systolic tension generation, and impairs relaxation in a $\mathrm{Ca}^{2+}$-independent manner. Elevated expression of the variant in the indirect flight muscles (IFMs) has dose-dependent effects, progressively impairing flight and inducing destructive hypercontraction due to excessive, myosin-dependent force generation. Sinusoidal analysis of mutant IFM fibers reveals increased $\mathrm{Ca}^{2+}$ sensitivity of power development, while in vitro motility experiments indicate disinhibited M305L actin-Tpm filaments. Molecular dynamics (MD) simulations suggest that the flexibility and interconnectivity of $\mathrm{M} 305 \mathrm{~L}$ actin subregions are drastically reduced. Importantly, the amino acid stretch containing K326, $\mathrm{K} 328$, E334, and a residue that protrudes out from the F-actin backbone, P333, likewise displays substantially restricted motion and aberrant intramolecular communication. This could disrupt the formation of electrostatic interfacial contacts and the azimuthal stability of inhibitory Tpm positioning, as well as the unimpeded translocation of Tpm over the thin filament surface. Finally, computational chemistry predicts a poorly maintained Astate of M305L actin-Tpm filaments as reflected by a diminution of the energy basin that biases Tpm to an inhibitory configuration. In vivo, this lack of inhibitory bias likely impacts the B-state and accounts for the impaired regulatory behavior of Tncontaining thin filaments. Thus, our models recapitulate the earliest signs of HCM, including impaired relaxation and hyperdynamic contractile properties ${ }^{3}$. Furthermore, our findings are indicative of a gain-in-sarcomeric-function that results from a reduction in actin flexibility and concurrent destabilization of Tpm positioning along, and impaired movement over, thin filaments, which in humans may trigger HCM remodeling events.

\section{Results}

M305L actin induces cardiomyopathy in Drosophila. Flies, like vertebrates, express specific actin isoforms in different muscles (Supplementary Fig. 2). Act57B is one of two sarcomeric actin genes expressed in the adult fly heart, while Act $88 F$ encodes all sarcomeric actin in the IFM ${ }^{32-34}$. The Drosophila heart consists of a single layer of bilateral rows of cardiomyocytes that form a simple linear tube (Fig. 1a) ${ }^{35}$. To determine if ectopically expressed M305L actin properly folds and incorporates evenly along Drosophila cardiac thin filaments, Hand ${ }^{4.2}$-Gal4-expressing virgin female flies were crossed with males carrying a transgene comprised of an upstream activating sequence (UAS) followed by GFPlabeled $A c t 57 B$. The progeny (abbr. Hand $>A c t 57 B^{G F P . W T}$ and Hand $\left.>A c t 57 B^{G F P . M 305 L}\right)$ inherit both genes and express GFPactin exclusively in the heart (Fig. 1a, b). At high $(\times 100)$ magnification, we observed Act57BGFP.M305L actin incorporating and extending uniformly along the entire thin filament in a manner 


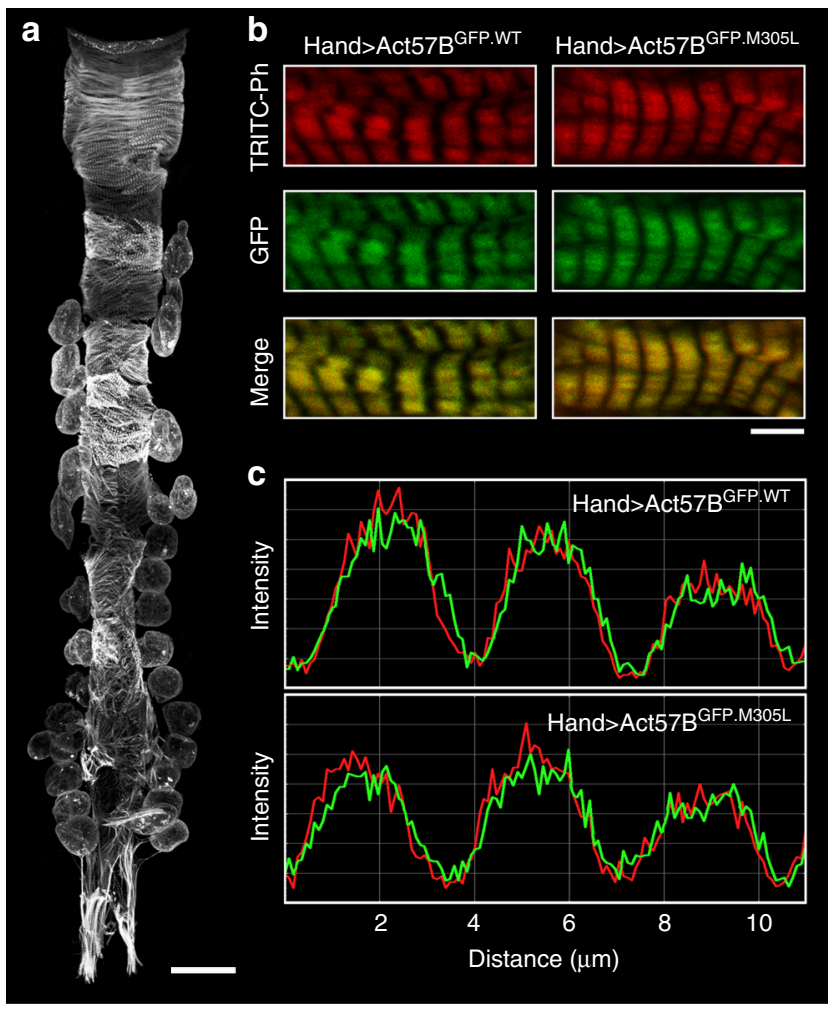

Fig. 1 M305L actin incorporates evenly along Drosophila cardiac thin filaments. a Composite confocal image of a full-length Hand $>$ Act57BGFP.WT adult Drosophila heart tube. Four micrographs were acquired at $\times 40$ magnification and computationally stitched together. Scale bar $=100 \mu \mathrm{m}$. b Fluorescent signals from Act57BGFP.WT and Act57BGFP.M305L actin, within fly cardiomyocytes, acquired at $\times 100$ magnification. The GFP signals co-localized repetitively with TRITC-phalloidin (TRITC-Ph) signals. TRITCPh labels both transgenic and endogenous cardiac actin. Scale bar $=5 \mu \mathrm{m}$. c Fluorescent intensity line scans of sarcomeres in (b) reveal overlapping and congruent TRITC-ph (red) and GFP (green) signals, indicating that Act57BGFP.M305L mutant actin copolymerized with endogenous cardiac actin along the length of the thin filaments, similar to control Act57B GFP.WT transgenic actin.

that was indistinguishable from Act57BGFP.WT actin (Fig. 1b). The GFP signals co-localized with TRITC-phalloidin signals, indicating homogenous co-polymerization of endogenous and transgenic actin isoforms (Fig. 1c). Act57B GFP.M305L did not degrade or aggregate, nor was it excluded from the sarcomere. Importantly, quantitative western blot analysis confirmed that Hand ${ }^{4.2}$-Gal4 drove comparable amounts of wildtype and mutant transgenic actin expression, with each comprising roughly $10 \%$ of total cardiac actin, in the respective fly hearts (Supplementary Fig. 3A).

To decipher the consequences of the HCM actin mutation on Drosophila cardiac performance, we evaluated beating hearts of female flies expressing UAS-Act57BWT or UAS-Act57B $B^{M 305 L}$ transgenes that lacked the N-terminal GFP moiety, which our lab has shown can confound results ${ }^{31}$. M-Mode kymograms, generated using high-speed video microscopy and semiautomated optical heartbeat analysis software ${ }^{36,37}$, suggested cardiac-restricted expression of the actin variant reduced heart tube diameters, prolonged systole, and reduced the rate of myocardial relaxation relative to control (Fig. 2a). We quantified these and additional indices of cardiac function and assessed potential genotype- and dose-dependent effects by employing multiple cardiac-specific GAL4 driver lines and distinct experimental conditions (Fig. 2b-f; Supplementary Fig. 3B, C). The latter entailed altering the rearing temperature, which results in a range of expression levels of UAS responder transgenes ${ }^{38}$. Based on the use of a nuclear-specific UAS-GFP reporter, the 4XHand- (at $25^{\circ}$ C) and TinC-Gal4 (at $29^{\circ} \mathrm{C}$ ) drivers were expected to yield $\sim 4$-fold higher $U A S-A c t 57 B^{W T}$ or $U A S-A c t 57 B^{M 305 L}$ expression levels in cardiomyocytes relative to Hand ${ }^{4.2}$-Gal4 (at $25^{\circ} \mathrm{C}$ ) (Supplementary Fig. 3B). Regardless of the driver or temperature, UAS$A c t 57 B^{M 305 L}$ actin expression significantly diminished cardiac output relative to controls, which was primarily due to restricted diastolic and systolic diameters coupled with significantly decreased fractional shortening (Fig. 2b-d, Supplementary Table 1). Flies expressing UAS-Act57B ${ }^{M 305 L}$ exhibited a significantly higher SI/HP ratio, which represents the proportion of time spent during the cardiac cycle generating systolic tension, and significantly decreased relaxation rates (Fig. 2e, f). The relative, physiological differences between control and mutant hearts were not exacerbated when M305L actin was expressed at higher doses using the stronger $4 \mathrm{XHand}$-Gal4 or TinC-Gal4 $\left(\right.$ at $29^{\circ} \mathrm{C}$ ) drivers (Supplementary Fig. 3C). Thus, comparable to the functional changes associated with human HCM, M305L actin expression in the fly heart, irrespective of genetic background, resulted in hyperdynamic contractile properties and it impaired relaxation and the ability to reestablish resting diastolic volumes.

HCM is frequently characterized by altered $\mathrm{Ca}^{2+}$ handling and elevated diastolic $\mathrm{Ca}^{2+}$ levels, which promote actomyosin associations and impair relaxation. To determine the mechanism of restricted cardiac diameters and to rule out a potential contribution from disrupted $\mathrm{Ca}^{2+}$ homeostasis, we incubated beating Hand $>A c t 57 B^{W T}$ and Hand $>A c t 57 B^{M 305 L}$ hearts in a solution containing $10 \mathrm{mM}$ EGTA and $100 \mu \mathrm{M}$ EGTA,AM ${ }^{31,39}$. EGTA-EGTA,AM chelates both extra- and intracellular $\mathrm{Ca}^{2+}$ and arrests contraction. Relative to that established during diastole, the heart tubes experienced a slight, yet significant, $~ 2 \%$ increase in diameters upon EGTA-EGTA,AM exposure (Fig. 2g). Thus, small amounts of residual intracellular $\mathrm{Ca}^{2+}$ are likely present in the hearts' of both genotypes during diastole that stimulate actomyosin interactions and produce slightly contracted cardiomyocytes. However, there was no difference in the average change in cardiac diameters between Hand $>A c t 57 B^{W T}(\Delta=1.53 \pm 0.15$ $\mu \mathrm{m})$ and Hand $>\operatorname{Act5} B^{M 305 L}(\Delta=1.48 \pm 0.15 \mu \mathrm{m})$ upon treatment (Fig. 2h). This suggests that a similar diastolic $\mathrm{Ca}^{2+}$ level exists among both genotypes, which contributes equally to resting tension, and the significantly restricted $A c t 57 B^{M 305 L} L_{\text {-expressing }}$ cardiac tubes do not result from elevated resting $\mathrm{Ca}^{2+}$. Unaltered $\mathrm{Ca}^{2+}$ handling was also supported by qPCR analysis of mRNA abundance (Supplementary Fig 4), which revealed no significant differences in transcript levels of L-type $\mathrm{Ca}^{2+}$ channels, ryanodine receptors, SERCA, $\mathrm{Na}^{+} / \mathrm{Ca}^{2+}$ exchangers, or in $\mathrm{IP}_{3}$ receptors between the lines.

Blebbistatin inhibits myosin and its addition to EGTA-EGTA, AM-treated fly hearts has been shown to elicit further increases in cardiac dimensions ${ }^{31,40,41}$. We confirmed blebbistatin's effect on Hand $>A c t 57 B^{W T}$ and Hand $>A c t 57 B^{M 305 L}$ heart diameters (Fig. 2g, i), which suggests that chelation of extra- and intracellular $\mathrm{Ca}^{2+}$ does not induce complete relaxation of Drosophila cardiomyocytes due to a small population of S1 cross-bridges that continue to bind thin filaments. To evaluate the relative proportion of thin filament-bound, $\mathrm{Ca}^{2+}$-independent, diastolic cross-bridges present in Hand $>A c t 57 B^{W T}$ vs. Hand $>A c t 57 B^{M 305 L}$ hearts, we compared the cardiac responses to blebbistatin treatment. The blebbistatin-induced increase in diameter for Hand $>A c t 57 B^{W T}$ heart tubes was $\sim 2.7 \%(\Delta=2.10 \pm 0.15 \mu \mathrm{m})$, while that for Hand > Act57 $B^{M 305 L}$ was $\sim 4.3 \%(\Delta=3.13 \pm 0.17 \mu \mathrm{m})$ (Fig. 2i). Blebbistatin had a significantly greater effect on Hand $>A c t 57 B^{M 305 L}$ hearts relative to controls. This result is consistent with diastolic dysfunction and restrictive physiology that is independent of 

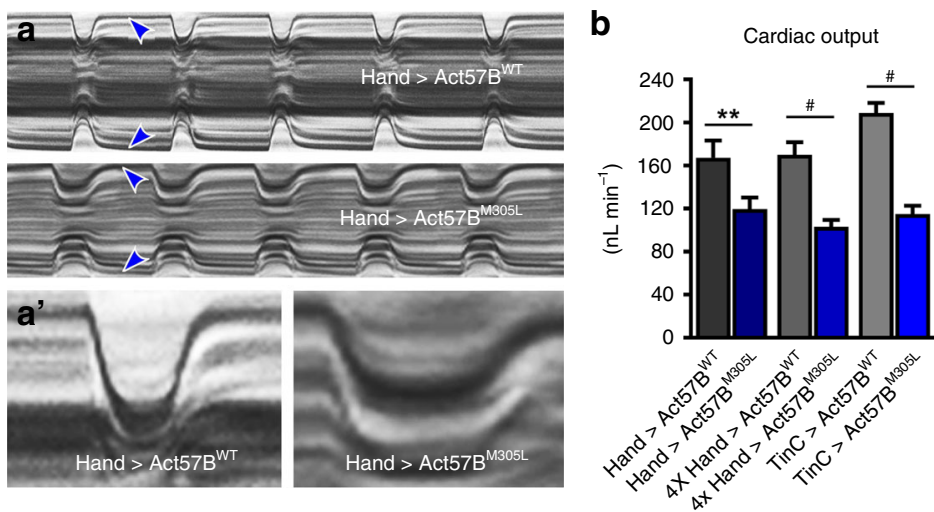

c

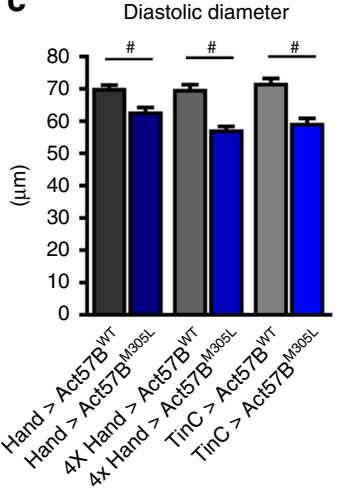

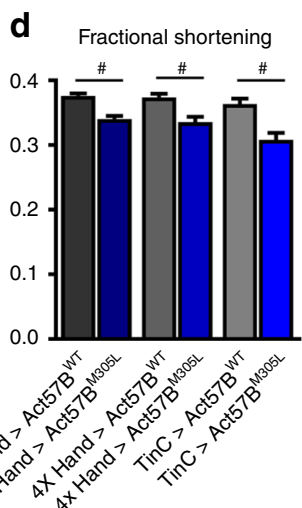

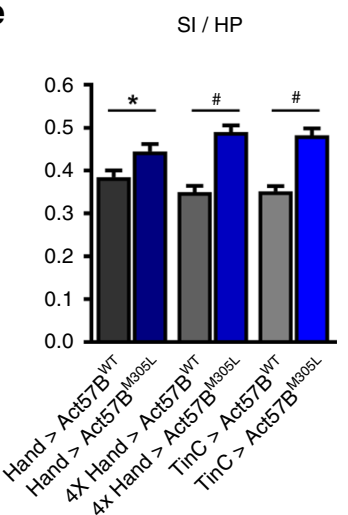

$\mathbf{f}$

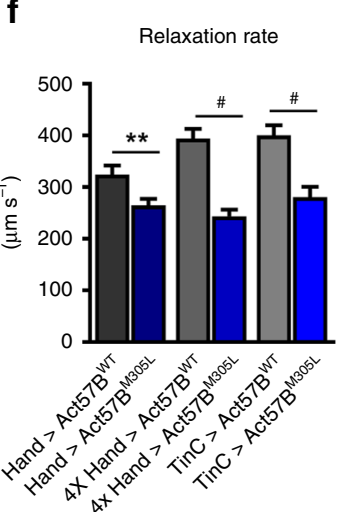

g
Cardiac diameters

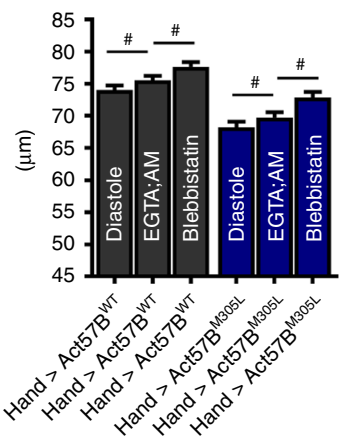

h Response to

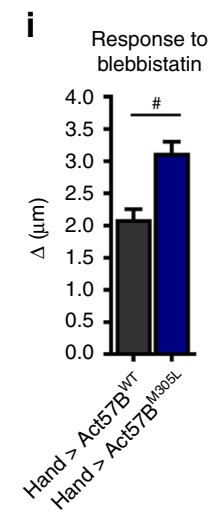

Fig. 2 M305L actin triggers restrictive cardiac physiology and impairs relaxation. a M-mode kymograms generated from high-speed videos of beating, three-week-old Hand $>A c t 57 B^{W T}$ and Hand $>A c t 57 B^{M 305 L}$ hearts. These traces illustrate cardiac cycle dynamics and heart wall motion over time. Blue arrowheads demarcate the edges of the heart wall during diastole. Note the restricted diastolic diameter across the mutant cardiac tube. $\mathbf{a}^{\prime}$ Individual systolic intervals taken from the traces shown in (a). Relative to Hand $>A c t 57 B W T$, Act57BM305L-expressing cardiac tubes exhibited prolonged periods of tension generation, diminished shortening, and slower relaxation rates. b-f Heart-restricted expression of Act57B ${ }^{M} 305 L$ mutant actin significantly altered several indices of cardiac function, irrespective of genetic background, relative to the expression of Act57BWT. Hand >, 4XHand >, and TinC > Act57BM305L Drosophila displayed decreased cardiac output, diastolic diameters, fractional shortening, and relaxation rates in addition to extended systolic periods relative to controls. SI/HP is the systolic interval over the time required for a complete cardiac cycle (i.e. diastolic plus systolic intervals). Significant differences between genotypes were determined using unpaired two-tailed $t$-tests $(n=31-45) .{ }^{\star} P \leq 0.05,{ }^{\star \star} P \leq 0.01$ and ${ }^{\#} P \leq 0.0001$. $\mathbf{g}$ Significant, incremental increases in cardiac diameters were observed in Hand $>A c t 57 B^{W T}$ and Hand $>A c t 57 B^{M} 305 L$ Drosophila following extra- and intracellular Ca $2+$ chelation and, again, upon blebbistatin exposure. Increases in cardiac dimensions due to EGTA-EGTA,AM and to blebbistatin were evaluated using repeated measures ANOVAs followed by Tukey's multiple comparison tests of the matched groups $(n=30-31)$. ${ }^{\#} P \leq 0.0001$. $\mathbf{h}$ The change in cardiac diameter in response to EGTA-EGTA,AM was similar between Hand $>A c t 57 B W T$ and Hand $>A c t 57 B^{M 305 L}$ flies as determined by two-tailed unpaired $t$-tests $(n=30-31)$. i Blebbistatin treatment resulted in a significantly greater degree of heart wall relaxation in Hand $>$ Act $57 B^{M} 305 L$ hearts relative to Hand $>$ $A c t 57 B^{W T}$ hearts. Two-tailed unpaired $t$-tests were used to distinguish significant differences in cardiac diameter changes between genotypes $(n=30-31)$. $\# P \leq 0.0001$. All data are presented as mean \pm SEM. Source data are provided as a Source Data file.

resting $\mathrm{Ca}^{2+}$ levels. Thus, the phenotype of Hand $>A c t 57 B^{M 305 L}$ mutant hearts is likely caused by excessively disinhibited, $\mathrm{Ca}^{2+}$ independent cross-bridge-binding to poorly regulated thin filaments, which results in enhanced basal tension and incomplete relaxation during diastole.

M305L actin impairs flight and promotes IFM hypercontraction. Drosophila IFMs are well suited for structural and mechanical analyses and are highly sensitive to sarcomeric perturbations, making them ideal for studying the phenotypic and functional effects of muscle protein mutations $31,39,42-46$. We created $A c t 88 F^{W T}$ and $A c t 88 F^{M 305 L}$ flies that expressed transgenic and endogenous actin at different ratios by backcrossing the transgene into a flightless, Act $88 F$-null strain (see Materials and Methods). Act88FM305L/ f flies, which express one transgenic and one endogenous copy of actin, exhibited a significant decrease in flight ability compared to $A c t 88 F^{W T} /+$ controls (Table 1). Despite flight differences, the IFMs of both lines appeared normal, each with six undisturbed dorsal longitudinal muscle fibers (DLMs) spanning the length of the thorax (Fig. 3a). When the ratio of transgenic to endogenous actin was increased to two to one, the flight ability of both the wildtype and mutant lines was diminished; however, the impairment observed for $A c t 88 F^{M 305 L} /$ Act88F $3305 \mathrm{~L}$; + was significantly greater than that for Act88FWT/ $A c t 88 F^{W T}$; + flies (Table 1). Nonetheless, the majority of Act88F $3305 \mathrm{~L} /$ Act88F $305 \mathrm{~L}$; Drosophila had intact IFMs with only a small percentage of flies displaying minor disturbances and breaks in their DLMs (Fig. 3a, Supplementary Fig. 5A). Homozygous expression of wildtype transgenic actin in $A c t 88 F^{W T}$ / $A c t 88 F^{W T}$, in an endogenous IFM-actin null background, did not rescue the flightless phenotype associated with the Act88F-null strain (Table 1), potentially due to insufficient transgene expression $^{31}$. Act $88 F^{W T} / A c t 88 F^{W T}$ IFM morphology, however, was phenotypically normal. Act88F $F^{M 05 L} /$ Act88F $F^{M 05 L}$ Drosophila were similarly flightless (Table 1), yet their IFMs exhibited 
destructive hypercontraction with $100 \%$ penetrance (Fig. 3a). This phenotype can result from excessive and/or unregulated force generation due to Tpm-based disinhibition of actomyosin interactions ${ }^{40,44}$. Thus, unlike what was observed in the fly heart, the IFM showed progressively worse phenotypes with increased M305L actin expression.

Since the M305L mutation has been shown to reduce F-actin lengths in vitro ${ }^{10}$, and thin filament length is an important determinant of force production in vivo ${ }^{47}$, we determined whether a mutation-induced change in thin filament length might contribute to M305L-mediated IFM pathology. The average thin filament lengths, measured from confocal micrographs of TRITC-phalloidin labeled IFM myofibrils from twoday-old Act $88 F^{W T} /+(1.40 \pm 0.01 \mu \mathrm{m}), A c t 88 F^{M 305 L} /+(1.38 \pm$ $0.01 \mu \mathrm{m}), A_{c t 88 F^{W T}} / A c t 88 F^{W T} ;+(1.39 \pm 0.01 \mu \mathrm{m}), A^{2} 88 F^{M 305 L} /$ Act88FM305L; $\quad(1.37 \pm 0.01 \mu \mathrm{m}), \quad$ Act88FWT/Act88FWT $(1.39 \pm$ $0.01 \mu \mathrm{m})$, and $A c t 88 F^{M 305 L} / A c t 88 F^{M 305 L}(1.37 \pm 0.01 \mu \mathrm{m})$ Drosophila, were not significantly different (Fig. 3b, Supplementary Fig. 5B). Thus, disrupted thin filament length is likely not a contributing factor to Act $88 \mathrm{~F}^{\mathrm{M} 305 \mathrm{~L}}$-induced hypercontraction.

M305L actin enhances $\mathrm{Ca}^{2+}$ sensitivity of IFM fibers. At the cellular and molecular levels, HCM-causing thin filament

\section{Table 1 Flight indices of Drosophila expressing varying amounts of transgenic versus endogenous Act88F.}

\begin{tabular}{|c|c|c|c|}
\hline Genotype & Tg:Endo & Flight index & $\mathrm{n}$ \\
\hline Act88F $F^{W T} /+$ & $1: 1$ & $5.05 \pm 0.08$ & 206 \\
\hline Act88FM305L $/+$ & $1: 1$ & $3.85 \pm 0.11^{\#}$ & 212 \\
\hline Act $88 \mathrm{~F}^{W T} / \mathrm{Act} 88 \mathrm{~F}^{W T} ;+$ & $2: 1$ & $3.98 \pm 0.11$ & 199 \\
\hline Act88FM305L/Act88FM305L;+ & $2: 1$ & $1.74 \pm 0.08^{\#}$ & 227 \\
\hline Act88FWT/Act88FWT & $2: 0$ & $0.00 \pm 0.00$ & 222 \\
\hline Act88F $\mathrm{F}^{\mathrm{M} 305 \mathrm{~L}} / \mathrm{Act}_{88 \mathrm{~F}^{\mathrm{M}} 305 \mathrm{~L}}$ & $2: 0$ & $0.00 \pm 0.00$ & 222 \\
\hline
\end{tabular}

Tg:Endo refers to the ratio of transgenic to endogenous Act88F gene copy numbers. Data are presented as mean \pm SEM. \#Significantly different from the corresponding WT control line as determined by the Mann-Whitney test $(P \leq 0.0001)$. Source data are provided as a Source Data file. mutations are frequently associated with impaired contractile regulation as characterized by elevated $\mathrm{Ca}^{2+}$ sensitivity of activation $^{4,5}$. To ascertain the influence of the M305L actin mutation on $\mathrm{Ca}^{2+}$ sensitivity and mechanical properties of muscle cells, we conducted sinusoidal analysis of skinned IFM fibers. Drosophila IFM isometric tension is exceedingly low, especially at minimal and intermediate $\mathrm{Ca}^{2+}$ concentrations, which greatly compromises signal to noise ratio ${ }^{46}$. Therefore, IFM power and elastic modulus are more amenable to producing an accurate measurement of $\mathrm{Ca}^{2+}$ sensitivity. We determined the power generated in IFMs as a function of $\mathrm{Ca}^{2+}$ from heterozygous $A c t 88 F^{W T} /+$ and $A c t 88 F^{M 305 L} /+$ flies. Act $88 F^{W T} /+$ and Act $88 F^{M 305 L} /+$ IFM fibers were analyzed since they appeared overtly intact and structurally normal (Fig. 3a) and to best represent the dominant condition observed in afflicted patients. Act $88 \mathrm{~F}^{\mathrm{M} 305 \mathrm{~L}}$-containing fibers displayed increased $\mathrm{Ca}^{2+}$ sensitivity, as evidenced by a significant leftward shift in $\mathrm{pCa}_{50} \mathrm{com}$ pared to Act $88 \mathrm{~F}^{\mathrm{WT}}$ controls with no difference in maximum power generated (Fig. 3c, Table 2). The slope of the curve (Hill coefficient) was not significantly different between fiber types indicating no change in cooperativity of thin filament activation. Elastic modulus was measured at $500 \mathrm{~Hz}$ and also revealed a leftward shift in $\mathrm{pCa}_{50}$ in mutant fibers with no change in Hill coefficient or maximum elastic modulus (Supplementary Fig 5C and Supplementary Table 2). The shift in $\mathrm{Ca}^{2+}$ sensitivity was likely not due to changes in cross-bridge cycling kinetics, as the frequency at which maximum power was generated $\left(f_{\max }\right)$ was not significantly different between Act $88 \mathrm{~F}^{\mathrm{M} 305 \mathrm{~L}}$ and Act $88 \mathrm{~F}^{\mathrm{WT}}$ actin-containing fibers (Table 2). Similarly, muscle apparent rate constants $2 \pi \mathrm{b}$, primarily influenced by steps associated with myosin binding to actin, the power stroke and Pi release, and $2 \pi \mathrm{c}$, largely defined by the strongly bound steps including ADP release and ATP-induced myosin detachment from actin, were not significantly affected by the mutation.

M305L cardiac actin induces myosin-dependent IFM pathology. To determine whether misexpression of M305L cardiac actin in skeletal muscle would also elicit a hypercontractile phenotype, and if this potentially was myosin-dependent, $U A S-A c t 57 B^{W T}$ and
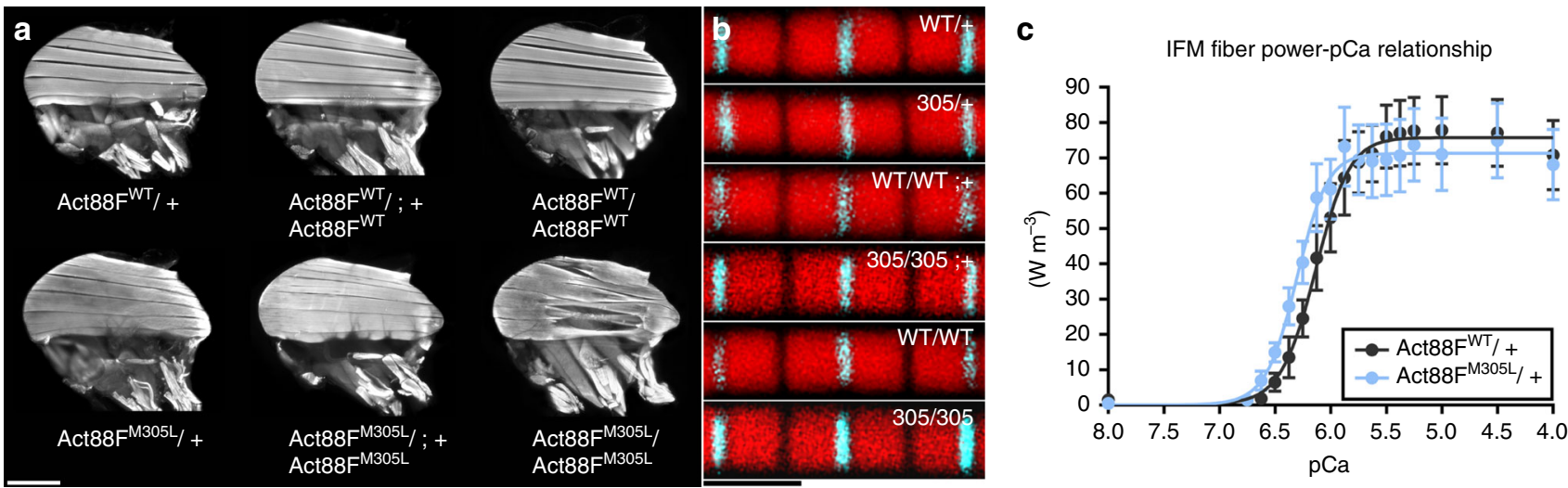

Fig. 3 M305L actin causes IFM hypercontraction and enhances $\mathbf{C a}^{\mathbf{2}+}$ sensitivity. a Fluorescent micrographs of dorsal longitudinal IFMs (DLMs) of twoday-old Act88FWT and Act88FM305L Drosophila. Act88FWT/+, Act88FM305L/+, Act88FWT/Act88FWT; +, Act88FM305L/Act88FM305L;+ heterozygotes, and

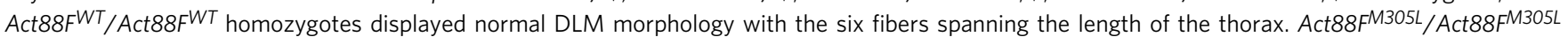
homozygotes, however, demonstrated hypercontracted and torn DLMs. Scale bar $=250 \mu \mathrm{m}$. $\mathbf{b}$ Confocal images of consecutive sarcomeres along a single IFM myofibril from transgenic flies. Red, TRITC-phalloidin-labeled actin; Cyan, immunolabeled $\alpha$-actinin. WT $/+=$ Act88FWT/+, 305/+ = Act88FM305L $/+$, WT/WT; $+=$ Act88FWT/Act88FWT; + , 305/305; $+=$ Act88FM305L/Act88FM305L; + , WT/WT $=$ Act88FWT/Act88FWT, and 305/305 = Act88FM305L/ Act88FM305L. IFM thin filament lengths did not significantly differ among the genotypes (Supplementary Fig. 5B) as determined by a Kruskal-Wallis oneway ANOVA with Dunn's post hoc test $(n=200-211)$. Scale bar $=2.5 \mu \mathrm{m}$. c The power-pCa relationship of Act88FM305L/+ IFM fibers revealed a significant leftward shift in $\mathrm{Ca}^{2+}$ sensitivity (see Table 2), indicating less $\mathrm{Ca}^{2+}$ is required for activation. Significance was assessed via an unpaired twotailed $t$-test $(n=10)$. Data points are mean \pm SEM and were fit by the Hill equation. Source data are provided as a Source Data file. 
Table 2 Power measurements and muscle apparent rate constants determined from IFM mechanical analysis.

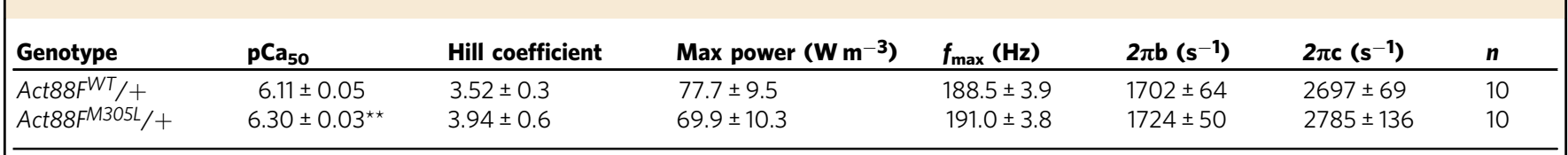

Data are presented as mean \pm SEM. ${ }^{* *}$ Significantly different from control Act88FWT/+ fibers as determined by Student's $t$ test $(P \leq 0.01)$. Source data are provided as a Source Data file.

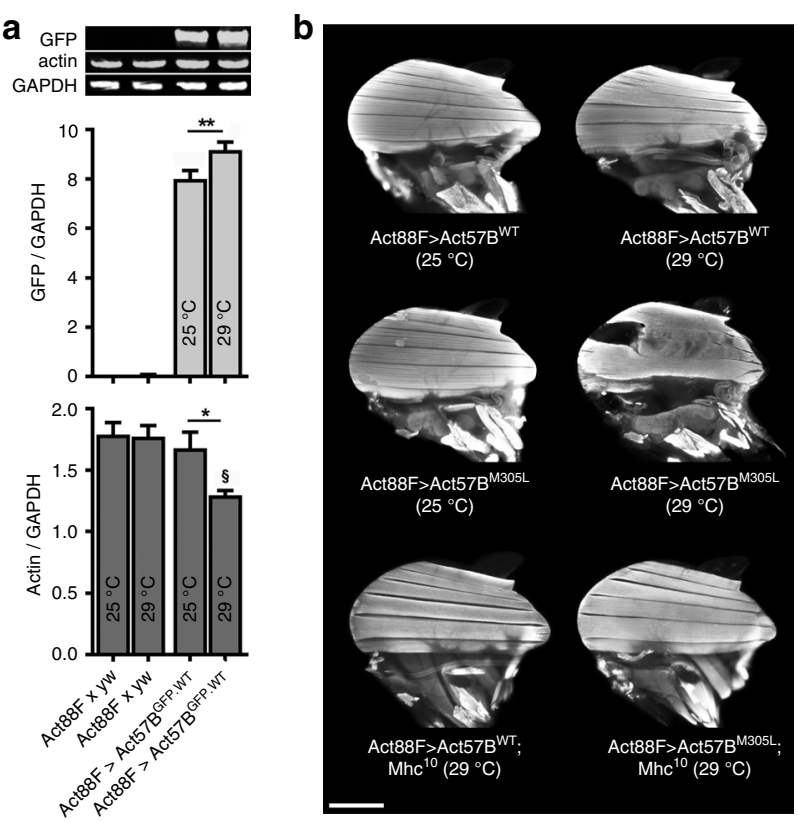

Fig. 4 High dose overexpression of M305L cardiac actin disrupts the IFM in a myosin-dependent fashion. a Quantitative western blot analysis of Act57B GFP.WT and endogenous actin was performed on IFMs from the progeny of Act $88 \mathrm{~F} \times \mathrm{yw}$ (control) and Act88F $>$ Act57BGFP.WT Drosophila raised at $25^{\circ} \mathrm{C}$ and $29^{\circ} \mathrm{C}$, two days after eclosion. Representative western blot, probed with antibodies that targeted GFP, actin, and GAPDH, showing expression of Act57BGFP.WT actin in Act88F $>$ Act57B GFP.WT flies and an absence of GFP-actin in control IFMs. The GFP-actin intensities (normalized to GAPDH) were significantly higher in flies raised at $29^{\circ} \mathrm{C}$. Actin intensities (normalized to GAPDH) revealed that Act88F > Act57BGFP.WT Drosophila raised at $29^{\circ} \mathrm{C}$ had a significant reduction in non-tagged, endogenous IFM actin. Quantification was performed on six independent biological replicates with three technical replicates each. Significance was assessed via one-way ANOVA with Tukey's multiple comparison test. ${ }^{\star} P \leq$ $0.05,{ }^{\star \star} P \leq 0.01 ; \$ P \leq 0.01$ relative to Act88F $x y w$ at $25^{\circ} \mathrm{C}$ or $29^{\circ} \mathrm{C}$. All data are presented as mean \pm SEM. Source data are provided as a Source Data file. b Fluorescent micrographs of dorsal longitudinal IFMs (DLMs) of two-day-old Act88F $>$ Act57BWT and Act88F $>$ Act57BM305L Drosophila. Act $88 \mathrm{~F}>A c t 57 B^{M 305 L}$ flies raised at $25^{\circ} \mathrm{C}$ displayed similar IFM morphology to Act88F $>$ Act57BWT flies. Conversely, Act88F $>$ Act57BM305L flies raised at $29^{\circ} \mathrm{C}$, with elevated mutant actin, showed hypercontracted IFMs, with the middle fibers pulling away from anterior attachment sites. A reduction in IFM myosin content, due to the presence of a single copy of the $\mathrm{MhC}^{10}$ (IFM-specific MHC null) allele, had no effect on the gross DLM morphology of Act88F $>$ Act57B ${ }^{W T} ; M_{10} 10 /+$ Drosophila (raised at $29^{\circ} \mathrm{C}$ ). Act88F $>$ Act57B M305L $^{\prime} M \mathrm{MhC}^{10} /+$ Drosophila (raised at $29^{\circ} \mathrm{C}$ ) displayed a complete rescue of the hypercontracted phenotype. Scale bar $=250 \mu \mathrm{m}$.

$U A S-A c t 57 B^{M 305 L}$ transgenes were expressed at variable doses with the Act88F-Gal4 IFM driver. The flies were raised at ambient $\left(25^{\circ} \mathrm{C}\right)$ or elevated $\left(29^{\circ} \mathrm{C}\right)$ temperature to increase protein load. The effect of elevated temperature on transgenic actin expression was confirmed by crossing Act88F-Gal4 females with UAS-Act57B ${ }^{G F P}$. $W T$ males; control flies were the progeny of Act88F-Gal4 females crossed with $y w$ males. IFMs from UAS-Act57BGFP.WT-driven and control flies, reared at $25^{\circ} \mathrm{C}$ or $29^{\circ} \mathrm{C}$, were dissected and subjected to quantitative western blot analysis using actin and GFP antibodies (Fig. 4a). GFP and actin signal intensities were measured and normalized to that from GAPDH. As expected, GFP was undetected in controls maintained at either temperature. IFMs from Act $88 \mathrm{~F}>$ $A c t 57 B^{G F P . W T}$ flies, however, had substantial amounts of GFP-actin, with elevated temperature inducing significantly higher expression relative to that at $25^{\circ} \mathrm{C}$. Transgenic cardiac GFP-actin comprised $\sim 20 \%$ and $\sim 30 \%$ of total IFM actin in flies maintained at $25^{\circ} \mathrm{C}$ and $29^{\circ} \mathrm{C}$, respectively (Supplementary Fig. 5D). Endogenous actin abundance was unaffected when Act88F $>U A S-A c t 57 B^{G F P . W T}$ flies were raised at $25^{\circ} \mathrm{C}$. Notably, increased expression of $A c t 57 B^{G F P . W T}$ coincided with a significant reduction in the amount of endogenous actin (Fig. 4a). This potentially indicates that there is a maximum level for actin overexpression in the IFM, beyond which stoichiometry is disrupted and compensatory downregulation of the endogenous isoform ensues.

Since GFP-labeled actin can induce flightlessness ${ }^{30,48}$, flight tests were performed on two-day-old $A c t 88 F>A c t 57 B^{W T}$ and $>A c t 57 B^{M 305 L}$ Drosophila. Low dose ectopic expression $\left(25^{\circ} \mathrm{C}\right)$ of cardiac UAS-Act57B $3305 L$ actin had no effect on flight ability relative to expression of $U A S-A c t 57 B^{W T}$ (Table 3), and fluorescence microscopy revealed normal IFM morphology (Fig. 4b). However, higher expression levels $\left(29^{\circ} \mathrm{C}\right)$ of $U A S-A c t 57 B^{W T}$ and $U A S-A c t 57 B^{M 305 L}$ actin resulted in a reduction and a loss of flight, respectively (Table 3 ). Act $88 F>A c t 57 B^{W T}$ Drosophila raised at 29 ${ }^{\circ} \mathrm{C}$ showed normal IFM structure while Act $88 F>A c t 57 B^{M 305 L}$ had damaged DLMs, with the middle fibers bunching and pulling away from their anterior attachment sites, indicative of hypercontracted muscle (Fig. 4b). Thus, independent of the actin isoform, the M305L amino acid substitution induced dose-dependent hypercontraction of skeletal muscle that did not support function.

To verify that the fiber tears and bunching in Act88F> $A c t 57 B^{M 305 L}$ flies reared at $29^{\circ} \mathrm{C}$ were myosin dependent, Act88F $>A c t 57 B^{W T}$ and $A c t 88 F>A c t 57 B^{M 305 L}$ females were crossed to $M h c^{10} / M_{h} c^{10}$ IFM myosin-null homozygous males to create progeny that express $A c t 57 B^{W T}\left(A c t 88 F>A c t 57 B^{W T} ; M h c^{10} /+\right)$ or $A c t 57 B^{M 305 L}\left(A c t 88 F>A c t 57 B^{M 305 L} ; M h c^{10} /+\right)$ in combination with a $50 \%$ reduction in myosin. Strikingly, Act $88 F>$ Act57B ${ }^{M 305 L} ; M_{h} c^{10} /+$ Drosophila raised at $29^{\circ} \mathrm{C}$ exhibited IFM morphology that was identical to that of $A c t 88 F>A c t 57 B^{W T}$; $M h c^{10} /+$ flies, with complete protection from M305L actininduced hypercontraction (Fig. 4b). This demonstrates that excessive myosin-dependent force generation is the underlying cause for fiber auto-destruction in Act $57 \mathrm{~B}^{\mathrm{M} 305 \mathrm{~L}}$-containing IFMs, and that the mutation plausibly disrupts the ability of the thin filament to properly regulate and oppose S1 cross-bridge binding.

M305L actin reduces Tpm-based inhibition of F-actin motility. Our results, obtained using both cardiac and skeletal muscle, collectively imply that the ACTC variant impairs Tpm-based steric blocking of S1 binding. Therefore, we directly measured the ability for the M305L mutation to upset Tpm positioning, and 


\begin{tabular}{|c|c|c|c|}
\hline Genotype & $\begin{array}{l}\text { Rearing } \\
\text { temp }\left({ }^{\circ} \mathrm{C}\right)\end{array}$ & Flight index & $n$ \\
\hline Act88F > Act57BWT & 25 & $5.21 \pm 0.08$ & 316 \\
\hline Act $88 \mathrm{~F}>A c t 57 B^{M 305 L}$ & 25 & $5.22 \pm 0.07$ & 408 \\
\hline Act $88 F>A c t 57 B W T$ & 29 & $2.74 \pm 0.12$ & 321 \\
\hline Act88F > Act57BM305L & 29 & $0.31 \pm 0.06^{\#}$ & 204 \\
\hline Act $88 \mathrm{~F}>A c t 57 B^{W T} ; M h c^{10}$ & 29 & $0.00 \pm 0.00$ & 103 \\
\hline $\begin{array}{l}\text { Act88F > Act57B } 1305 \text {; } \\
M h c^{10}\end{array}$ & 29 & $0.00 \pm 0.00$ & 114 \\
\hline \multicolumn{4}{|c|}{$\begin{array}{l}\text { Data are presented as mean } \pm \text { SEM. \#Significantly different from } A c t 88 F>A c t 57 B^{W T}\left(29^{\circ} \mathrm{C}\right) \text { at } \\
\text { determined by the Mann-Whitney test }(P \leq 0.0001) \text {. Source data are provided as a Source } \\
\text { Data file. }\end{array}$} \\
\hline
\end{tabular}

thus the inhibitory A-state, in vitro by quantifying the percent of Act $88 \mathrm{~F}^{\mathrm{WT}}$ vs. Act $88 \mathrm{~F}^{\mathrm{M} 305 \mathrm{~L}}$ filaments that were propelled by varying concentrations of myosin. Previous studies have shown that under low myosin loading concentrations, the velocity and/ or the percent of motile actin filaments were significantly reduced upon the addition of Tpm, consistent with an inhibitory Factin-Tpm configuration ${ }^{49-52}$. Homogenous populations of IFM actin were purified from $A c t 88 F^{W T} / A c t 88 F^{W T}$ and $A c t 88 F^{M 305 L} /$ $A c t 88 F^{M 305 L}$ flies, labeled with TRITC-phalloidin, and subjected to in vitro motility analysis. The M305L mutation had no effect on the percent of filaments that were propelled by myosin in the absence of Tpm (Table 4). Moreover, as found earlier ${ }^{11}$, both wildtype and mutant $\mathrm{F}$-actin displayed similar sliding velocities (Supplementary Fig 6), which suggests the M305L mutation does not overtly influence actomyosin associations and the disease mechanism involves additional, higher order regulatory components. Consistent with this hypothesis, in the presence of vertebrate cardiac Tpm, the mutation elicited a significant increase in the percent of motile Act $88 \mathrm{~F}^{\mathrm{M} 305 \mathrm{~L}}$ relative to Act $88 \mathrm{~F}^{\mathrm{WT}}$ filaments at all myosin concentrations tested (Table 4). These results indicate that the M305L substitution likely destabilizes the native, inhibitory F-actin-Tpm A/B-state.

M305L actin exhibits low flexibility during MD simulations. To investigate mutation-induced changes in structural properties and protein dynamics, which may provide insight into the mechanism of Tpm mispositioning as well as fundamental properties of thin filament biology, we performed MD simulations of wildtype and M305L human monomeric (G-actin) and F-actin structural models (Supplementary Table 3). First, triplicate, 200 ns classical MD (cMD) simulations were carried out to characterize the effects of the mutation on G-actin. Principal component analysis (PCA $)^{53}$ was employed to reduce the dimensionality of the cMD trajectories and resolve dominant and concerted conformational changes in the protomers (Fig. 5a, b, Supplementary movies 1-4). Most of the conformational space sampled during cMD simulations was represented by only a few principal components (PCs), with the first three PCs comprising 70 to $80 \%$ of the variance in atomic positional fluctuations. The dominant motions in wildtype and mutant G-actins primarily included flexion and extension movements of SD2 and 4 (PC1) around a hinge joint localized in the interdomain connection between SD1 and 3, and a rotational twisting of the proteins (PC2). Projecting the two PCs onto the G-actin structural models revealed markedly reduced flexibility of $\mathrm{ACTC}^{\mathrm{M} 305 \mathrm{~L}}$ relative to $\mathrm{ACTC} \mathrm{CT}^{\mathrm{WT}}$, as indicated by the decreased amplitude of structural rearrangements in the mutant, both along PC1 and PC2 (Fig. 5a, b). This was corroborated by decreased root-mean-square-deviations (RMSD) of $\mathrm{ACTC}^{\mathrm{M} 305 \mathrm{~L}}$
Table 4 Percent of motile wildtype and mutant actin-Tpm filaments determined from in vitro analysis.

\begin{tabular}{|c|c|c|c|c|}
\hline Filament type & \pm Tpm & $\begin{array}{l}\text { [Myosin] } \\
\left(\mu \mathrm{g} \mathrm{ml}^{-1}\right)\end{array}$ & $\begin{array}{l}\text { Filaments } \\
\text { moving (\%) }\end{array}$ & $n$ \\
\hline F-Act88FWT & - & 12.5 & $31.02 \pm 1.64$ & 22 \\
\hline F-Act88FM305L & - & 12.5 & $32.57 \pm 2.34$ & 21 \\
\hline F-Act88FWT & + & 12.5 & $14.96 \pm 1.49$ & 20 \\
\hline F-Act88FM305L & + & 12.5 & $21.17 \pm 2.11^{\star}$ & 21 \\
\hline F-Act88FWT & - & 50 & $68.11 \pm 1.51$ & 16 \\
\hline F-Act88FM305L & - & 50 & $68.00 \pm 2.30$ & 20 \\
\hline F-Act88FWT & + & 50 & $56.43 \pm 1.04$ & 21 \\
\hline F-Act88FM305L & + & 50 & $67.81 \pm 1.37^{\#}$ & 21 \\
\hline F-Act88FWT & - & 100 & $64.09 \pm 1.64$ & 19 \\
\hline F-Act88FM305L & - & 100 & $67.89 \pm 1.61$ & 22 \\
\hline F-Act88FWT & + & 100 & $62.87 \pm 1.83$ & 20 \\
\hline F-Act88FM305L & + & 100 & $71.16 \pm 1.78^{\star \star}$ & 22 \\
\hline
\end{tabular}

Data are presented as mean \pm SEM. ${ }^{\star} P \leq 0.05,{ }^{\star \star} P \leq 0.01,{ }^{\#} P \leq 0.0001$ Significantly different from $\mathrm{F}-$ Act88 $\mathrm{F}^{\mathrm{WT}}+\mathrm{Tpm}$. Significant differences between filament types $\pm \mathrm{Tpm}$ at each myosin concentration were assessed using a two-way ANOVA with Bonferroni post-tests. Source data are provided as a Source Data file.

subdomains, particularly of SD2 and SD4 (Supplementary Fig. 7A). Excluding the highly flexible DNase-binding loop (residues 39 to 50) and hydrophobic plug (residues 263 to 271), which were shown earlier to retain high mobility in monomeric and F-actin ${ }^{54}$, the backbone atoms of SD2 and SD4 of mutant Gactin exhibited RMSDs up to $3.2 \AA$ and $3.3 \AA$ respectively, over the cMD simulations, as compared to $5.6 \AA$ and $4.9 \AA$ of wildtype. Accordingly, SD2 and SD4 of ACTC ${ }^{\mathrm{M} 305 \mathrm{~L}}$ remained closer together, both near the active site (inner cleft: between residues G15 and D157) and the protein surface (outer cleft: between residues E59 and R206) (Supplementary Fig 7B, upper panel). The radius of gyration, a measure of protein compactness, was consequently reduced in $\mathrm{ACTC}^{\mathrm{M} 305 \mathrm{~L}}$ vs. ACTC ${ }^{\mathrm{WT}}$ (Supplementary Fig. 7B, lower panel). Moreover, relative to wildtype, both SD2 and SD4 of ACTC ${ }^{\mathrm{M} 305 \mathrm{~L}}$ showed decreased $\mathrm{C}_{\alpha}$ root mean square fluctuations (RMSF) along the most dominant protein motions (Fig. 5c), as well as overall lower flexibilities in cMD simulations (Supplementary Fig. 7C), and along PC1 and PC2 (Supplementary Fig. 7D). Rearrangements of SD1 and SD3 were less pronounced and fluctuated around $1.5 \AA$ and $2 \AA$ for both isoforms. Additional details regarding differences in actinnucleotide hydrogen bonding can be found in Supplementary Fig. 7E, F.

To further analyze the effect of the M305L mutation on overall structural dynamics of ACTC, and to exclude artifacts from insufficient sampling of the cMD simulations in the actin equilibrium state, we next performed duplicate $500 \mathrm{~ns}$ enhanced sampling MD simulations using accelerated MD (aMD) simulations ${ }^{55-58}$. The conformational changes of wildtype and mutant G-actin in the aMD simulations followed a dynamic trend similar to that uncovered by cMD. We computed free energy landscapes (FEL) for both actins along the projections of the first two PCs, i.e. along the largest structural changes in the proteins, by energetic reweighting of the combined aMD simulations using Maclaurin series expansion ${ }^{59}$. The FEL of ACTC ${ }^{\mathrm{WT}}$ spanned a wide conformational space along PC1 and PC2 (Fig. 5d and Supplementary Fig. 8A). It was characterized by several minima, indicating a number of low-energy substates of the protein separated by energy barriers of up to $\sim 2 \mathrm{kcal} \mathrm{mol}^{-1}$. In contrast, the FEL of $\mathrm{ACTC} \mathrm{M}^{\mathrm{M} 305 \mathrm{~L}}$ was more restricted with a single narrow energy basin (Fig. 5e and Supplementary Fig. 8B). Hence, ACTC ${ }^{\mathrm{M} 305 \mathrm{~L}}$ seems limited to one conformational state, indicative of drastically reduced flexibility (Supplementary Fig. 8C, D). 
a

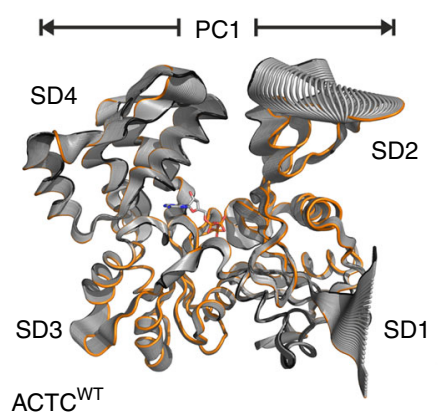

b

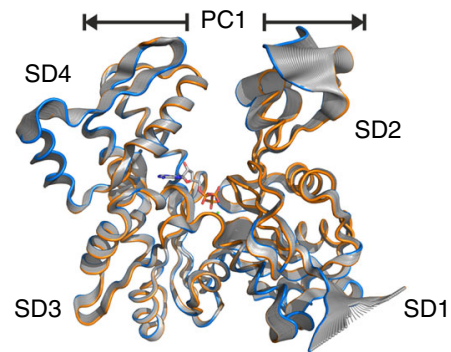

$\mathrm{ACTC}^{\mathrm{M} 305 \mathrm{~L}}$

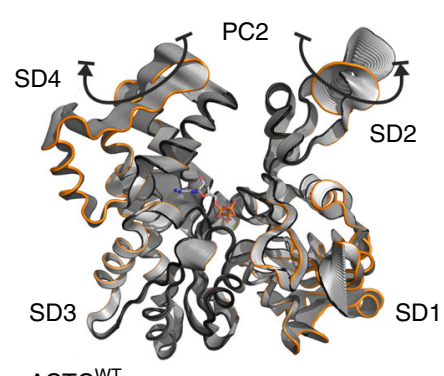

$\mathrm{ACTC}^{\mathrm{WT}}$

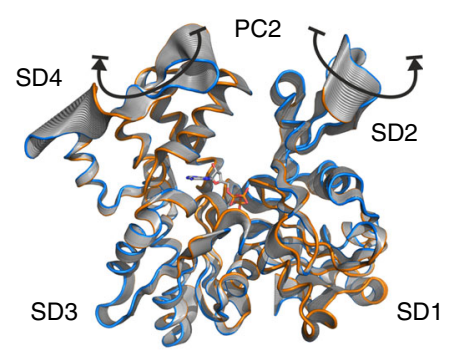

ACTC ${ }^{\text {M305L }}$

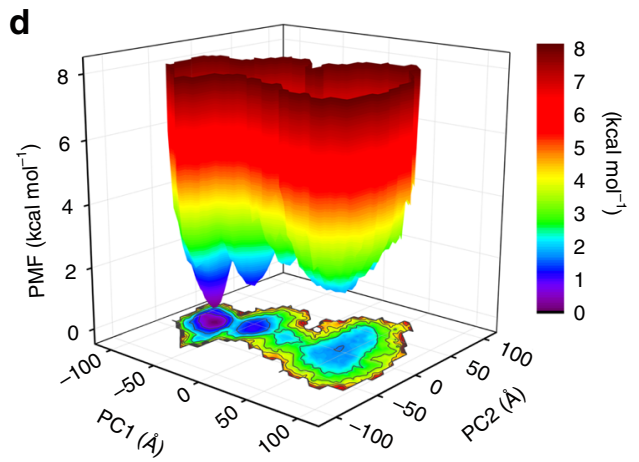

e

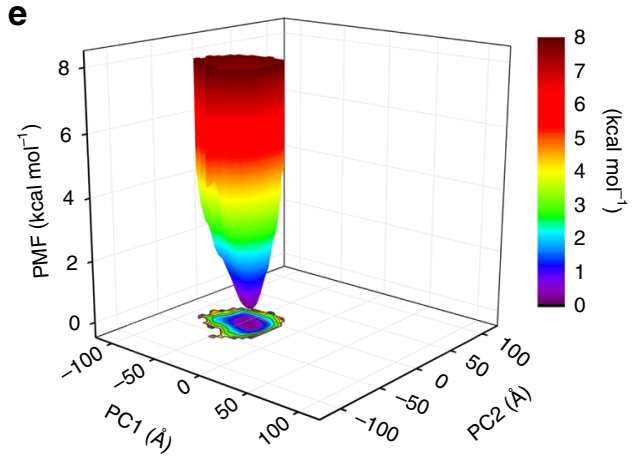

C
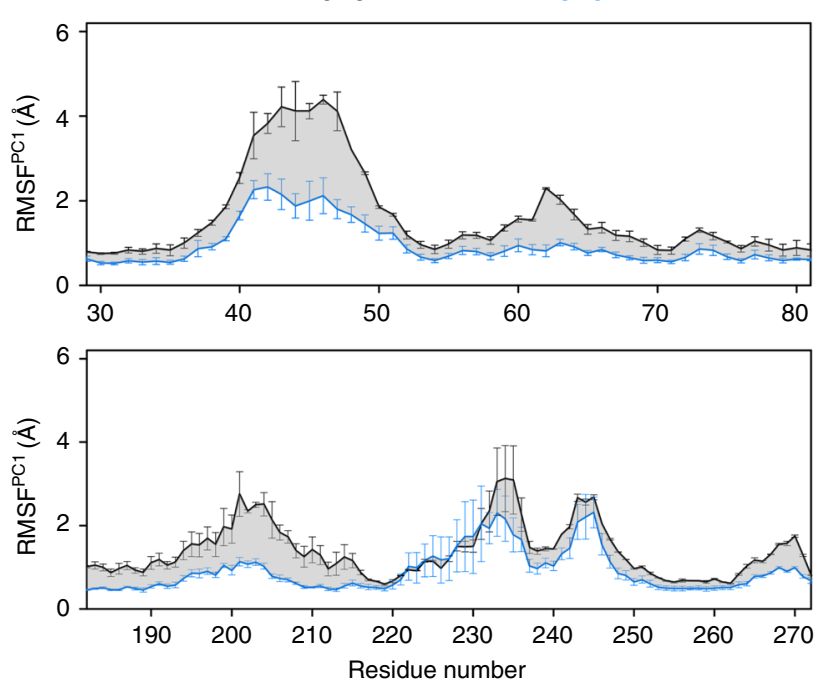

f
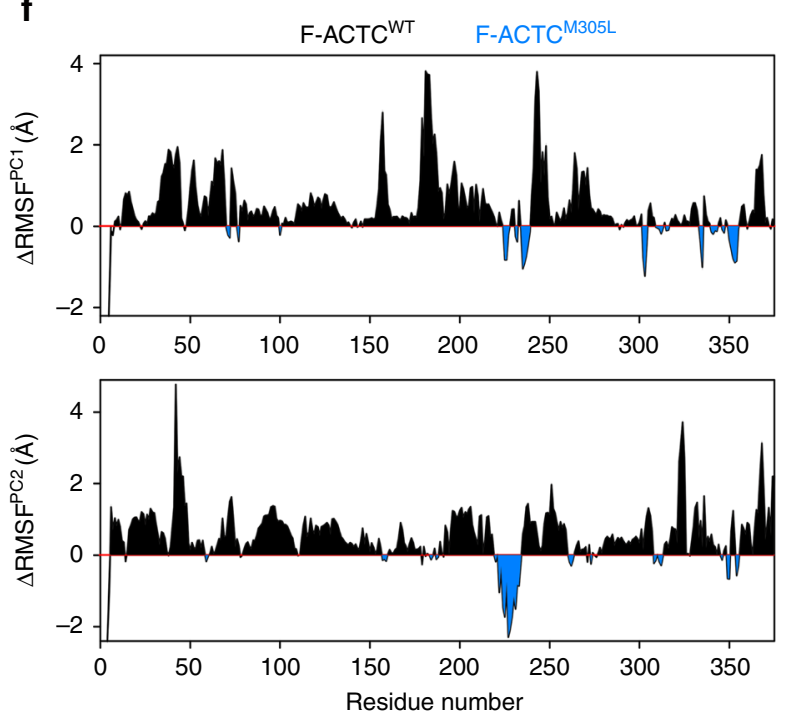

Fig. 5 The M305L mutation decreases actin flexibility in MD simulations. a Projections of the largest structural fluctuations according to the first principal component (PC) (left panel) and the second PC (right panel) of a representative cMD simulation of an ACTCWT monomer. The projections

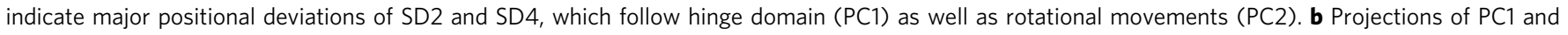
PC2 of a representative ACTCM305L monomer CMD simulation illustrate decreased protein motions compared to wildtype. c Root mean square

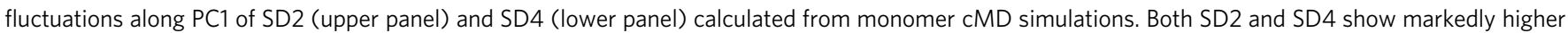
fluctuations in ACTCWT vs. ACTCM305L. Data are represented as mean \pm SEM $(n=3)$. d The free energy landscape of ACTCWT, calculated as the potential of mean force (PMF) according to the largest structural motions in the protein, as derived from PCA of two combined 500 ns enhanced sampling

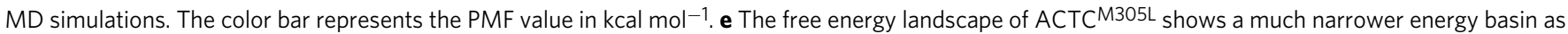

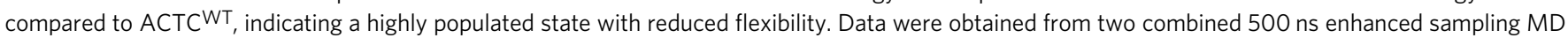
simulations. The color bar represents the PMF value in $\mathrm{kcal}^{\mathrm{mol}}{ }^{-1}$. $\mathbf{f}$ Root mean square fluctuations obtained from $\mathrm{F}$-actin $\mathrm{cMD}$ simulations, given as the difference of ACTCWT_ACTCM305L, along PC1 (upper panel) and PC2 (lower panel) confirm that the major protomer structural fluctuations persist in the filamentous actin form, and they are decreased in mutant actin. Source data are provided as a Source Data file.

Previous MD simulations predicted that much of G-actin's flexibility is retained by F-actin protomers in three different filament models $s^{54}$. Therefore, we proceeded to examine the mutation's effect on intrinsic protein flexibility in a physiologically relevant, filamentous form of ACTC by comparing duplicate
$200 \mathrm{~ns}$ cMD simulations of fully solvated ACTCWT to ACTC $^{\mathrm{M} 305 \mathrm{~L}} \mathrm{~F}$-actin. The hinge (PC1) and rotational (PC2) motions were also found in F-actin protomers and again represented the dominant structural changes (Supplementary Fig. 9). Consistent with the results of the monomer simulations, 
the overall protein flexibility, and particularly the RMSFs of SD2 and SD4, were significantly reduced in F-ACTC ${ }^{\mathrm{M} 305 \mathrm{~L}}$ as compared to wildtype along PC1 and PC2 (Fig. 5f), which suggests the effect of the actin mutation persists post polymerization.

\section{Assessment of protein allostery and F-actin-Tpm interaction.} Structural changes at distant actin sites can modulate various processes remotely via allosteric communication. Therefore, to further investigate the impact of the M305L substitution, including propagated effects, we performed network analysis of the ACTC MD trajectories (Supplementary Fig. 10A-D). Dynamical network analysis constructs network models by weighting interconnectivity within proteins according to the degree of correlated motions during simulations ${ }^{60}$. As expected, K326 and $\mathrm{K} 328$, which function to help confine Tpm to an inhibitory location along the F-actin surface ${ }^{22,24}$, were found within the same network community (dark blue) (Fig. 6a). In wildtype actin this community was interconnected with an adjacent, independent community that centered on P333 and E334 (brown). Note, $\mathrm{P} 333$, which projects out from the F-actin backbone, was recently suggested to form a flexible ridge that topologically divides the Band C-state Tpm positions along thin filaments and may, therefore, represent a crucial determinant of Tpm translocation during regulatory switching ${ }^{14}$. The mutation led to specific changes in the network communities and intercommunity communication (Fig. 6B and Supplementary Fig 10B). For instance, in close proximity to L305, several critical network connections observed in $\mathrm{ACTC} \mathrm{WT}^{\mathrm{T}}$ (green connections) were lost, indicating reduced communication in the vicinity of the lesion. The P333- and E334containing loop no longer moved individually, as found with wildtype actin (Fig. 6a), but was included in the community bearing the mutation (light blue) (Fig. 6b). Furthermore, comparing the isoforms' allosteric signaling pathways via crosscorrelation analysis, revealed altered signal propagation throughout ACTC ${ }^{\mathrm{M} 305 \mathrm{~L}}$ (Supplementary Fig 10e). Finally, based on the decidedly comparable backbone fluctuations previously noted during MD simulations of wildtype G- and multiple F-actin models ${ }^{54}$, the surface stretch of amino acids containing K326, K328, and E334, which form highly favorable, A/B-state-promoting contacts with Tpm, as well as P333, is expected to exhibit similar and relatively high mobility regardless of actin polymerization status. The M305L substitution however, distinctly reduced flexibility of this subregion (Fig. 6c). The ACTC mutation therefore, appears to decrease overall actin flexibility in the monomeric and filamentous forms, and it disturbs communication between protein subdomains, among regions in the active site, and with residues critical for Tpm interaction and inhibitory positioning along thin filaments.

Given the enhanced contractile and impaired relaxation properties of the mutant fly muscles, the increased motility of M305L actin-Tpm filaments, and the results of our MD simulations, we postulated that the molecular basis of Tpm's diminished ability to properly inhibit S1 binding could be due to reduced actin flexibility and subregion interconnectivity. Protomer flexibility and precise correlated subregion motion may be required to ensure that the complementary F-actin-Tpm interfacial surfaces and favorable electrostatic interactions persist in order to appropriately confine Tpm to an inhibitory location. Thus, reduced flexibility and intercommunity communication could potentially favor ACTC conformational substates that destabilize the interactions required for A/B-state Tpm positioning along F-actin. The average, composite electrostatic energy map of wildtype F-actin-Tpm, calculated from five individual landscapes using the most predominant $\mathrm{ACTC}{ }^{\mathrm{WT}}$ conformations extracted from cMD simulations, showed a single, deep, broad energy well that would ideally position Tpm to effectively deter S1 binding (Fig. 6d). In contrast, an electrostatic energy landscape similarly computed for $\mathrm{M} 305 \mathrm{~L}$ F-actin-Tpm revealed two prominent, discretely located basins. Each well was relatively narrow with roughly equivalent minima that had higher energies compared to wildtype. Inspection of the individual wildtype F-actin-Tpm electrostatic energy landscapes used to create the average landscape, revealed energy wells with minima displaying low positional variation that would consistently place Tpm very close to its typical, energetically optimal, inhibitory A/B-state location (Supplementary Fig. 11A). The M305L F-actin-Tpm energy landscapes however, were characterized by additional new, local minima with a high degree of positional variation (Supplementary Fig. 11B). Therefore, Tpm would not exclusively prefer the A/B-state energy basin and, consequently, may not be constrained to a single, inhibitory location along M305L filaments. Tpm is predicted to exhibit less positional bias and display a larger degree of azimuthal and longitudinal freedom around the M305L F-actin helix, which would result in improper inhibition of actomyosin associations and potentially stimulate human disease.

\section{Discussion}

HCM is characterized by myocardial hypertrophy, impaired relaxation, and hyperdynamic contractile properties ${ }^{1-3}$. The M305L ACTC HCM-causing mutation has been the subject of several in vitro studies, which have yielded both consistent and contradictory results ${ }^{8-11}$. A lack of congruent findings when using only actin, or actin and myosin, calls into question disease mechanisms and suggests higher order regulatory components may be required to resolve HCM pathogenesis, yet the effects of the mutation on thin filaments replete with Tn-Tpm complexes, or on myocyte behavior, have remained untested. Therefore, to help determine potential emergent pathogenic properties, both in vitro and in vivo, we generated transgenic animals that express M305L actin. Our fly models permit integrative analysis of the variant in distinct striated muscles from the molecular through the organ levels. Although the Drosophila cardiac tube is relatively simple compared to the mammalian heart, its cellular structure and proteomic makeup are highly conserved and it exhibits pathological responses analogous to those found in higher organisms $31,32,39,40,45,61,62$. Additionally, Drosophila IFMs are extremely useful for structural and mechanical analyses of sarcomeric mutations and they provide adequate material for biochemical investigation $31,39,42,44-46$

When overexpressed in the fly heart, M305L cardiac actin incorporated evenly along the entire length of the thin filaments (Fig. 1). It was not found in discrete subcellular locations nor was it excluded from the sarcomere. Thus, consistent with earlier in vitro studies $^{9,10}$, the amino acid substitution does not appear to impact protein folding or stability in vivo. In the IFMs, even in homozygotes expressing 100\% mutant actin, M305L thin filament length was the same as that of controls (Fig. 3b). Therefore, regardless of potential discrepancies in actin polymerization properties or depolymerization rates 9,10 , thin filament formation and integrity are unaffected in mutant sarcomeres and, hence, are unlikely to contribute to altered contractile properties and pathology.

Compared to controls, M305L-expressing heart tubes of three distinct genetic backgrounds, consistently displayed prolonged periods of systolic tension generation (Fig. 2e). Additionally, restricted diastolic volumes and decreased relaxation rates were observed for all mutant genotypes (Fig. 2b-f). The poor relengthening characteristics of $\mathrm{M} 305 \mathrm{~L}$ cardiomyocytes are indicative of defective relaxation. Diastole is normally 

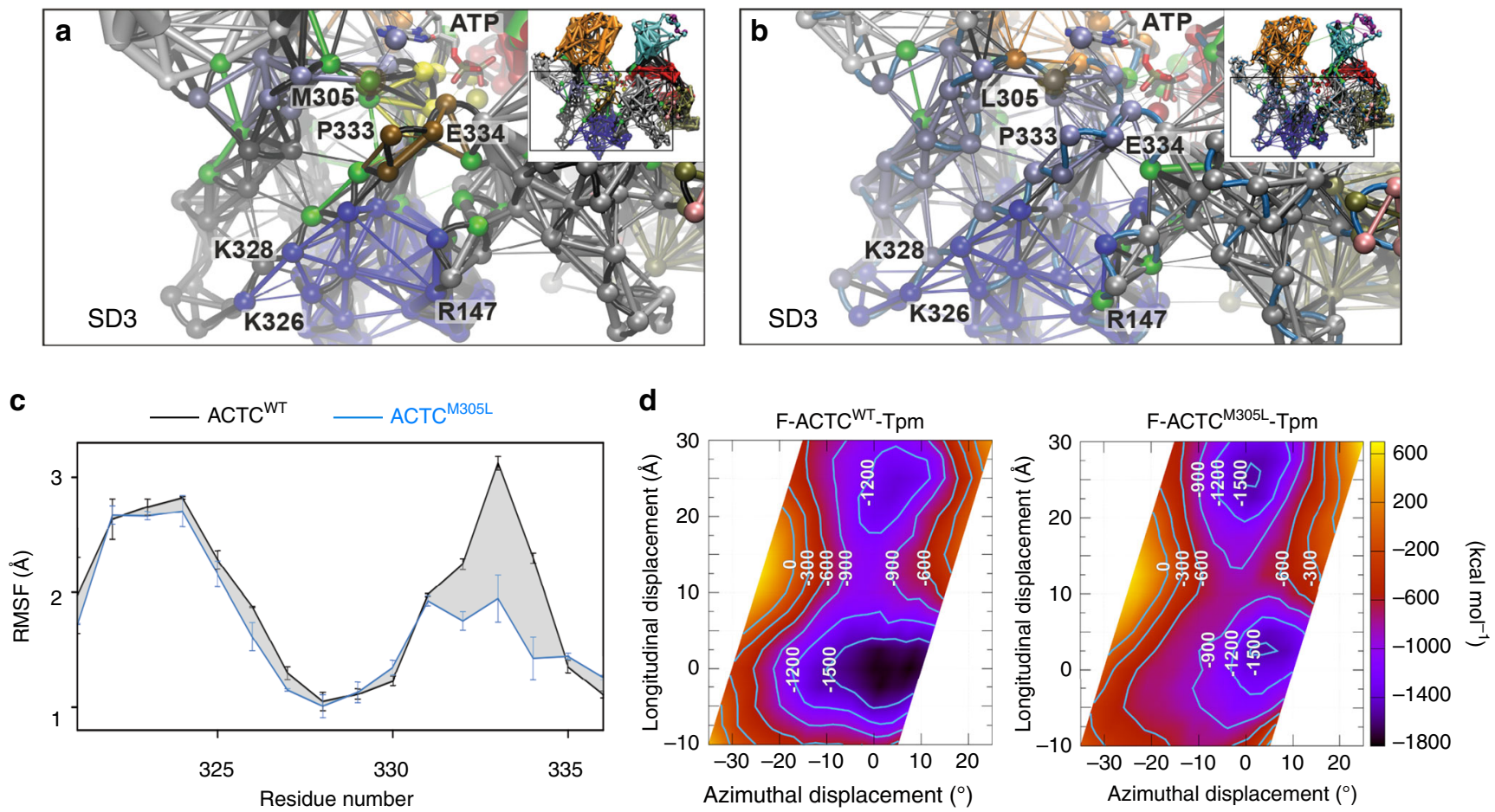

Fig. 6 ACTCM305L exhibits altered communication pathways and coupled motions and distorts F-actin-Tpm electrostatic energy landscapes. a

Dynamical network analysis of CMD simulations of an ACTCWT Tpm-interacting subregion revealed a high degree of coupled motions, represented by the thickness of the connections and weighted according to the correlation data obtained from PCA. Clustered network communities were identified using the Girvan-Newman algorithm and colored individually, indicating regions of concerted movements. Critical residues and connections between two network communities are presented in green. P333 and E334 formed a separate community (brown) that maintained some flexibility and moved independently with respect to K326 and K328. Inset: dynamical network analysis of an entire ACTCWT protomer (see Supplementary Fig. 10A) demarcating the enlarged region discussed above. $\mathbf{b}$ Dynamical network analysis of CMD simulations of ACTCM305L showed that the coupled motions of the Tpm-interacting subregion around P333 and E334 differed from those of ACTCWT and moved in concert with K326 and K328 and the community surrounding L305 (light blue). Inset: dynamical network analysis of an ACTCM305L protomer (see Supplementary Fig. 10B) demarcating the enlarged region discussed above. c Root mean square fluctuations (RMSF) of $C_{\alpha}$ atoms along a stretch of actin residues that forms stable interactions with and facilitates inhibitory positioning of Tpm, measured over the course of enhanced sampling MD simulations. Data are represented as mean \pm SEM ( $n=2)$. d Average electrostatic energy landscapes for wildtype (left) and M305L F-actin-Tpm (right). The origin is set at 0,0, which represents the previously determined energy minimum of the inhibitory, A/B configuration for wildtype F-actin-Tpm ${ }^{24}$, where Tpm is located in a position that would impede myosin binding. The plot is contoured with isolines between -1500 and $0 \mathrm{kcal} \mathrm{mol}^{-1}$ in increments of $300 \mathrm{kcal} \mathrm{mol}^{-1}$. Note, the broad well around the origin in the wildtype plot is shallower and narrower in the mutant F-actin-Tpm plot. Additionally, a second equivalent energy well is located distal to the A/B site along the mutant filament, which might be predicted to bias Tpm to a non-inhibitory location. Source data are provided as a Source Data file.

characterized by slightly shortened myocytes with modest basal tone $\mathrm{e}^{31,39,40,63}$ due to a limited number of $\mathrm{Ca}^{2+}$-dependent and $\mathrm{Ca}^{2+}$-independent actomyosin associations (Fig. $2 \mathrm{~g}$ ). However, comparing the changes in cardiac tube diameters in response to EGTA-EGTA,AM and then to blebbistatin suggested that the markedly shortened cardiomyocytes in latent mutant hearts were not caused by elevated diastolic $\mathrm{Ca}^{2+}$ levels, but rather resulted from poor Tpm-dependent blocking, and a disproportionately heightened number of disinhibited, actively cycling myosin cross-bridges (Fig. 2h, i). Irrespective of the actin isoform, the M305L mutation induced IFM hypercontraction, a phenotype previously shown to result from $\mathrm{Tpm}$ mispositioning 40,44 (Figs. 3a and $4 \mathrm{~b}$ ). The destructive syndrome was prevented by genetically ablating myosin (Fig. 4b). This implies that the mutation disrupts the thin filament B-state and permits excessive actomyosin cycling and force production that can be tempered by eliminating $50 \%$ of the motors. Finally, heterozygous Act88F ${ }^{M 305 L}$ IFM fibers exhibited enhanced $\mathrm{Ca}^{2+}$ sensitivity of power development (Fig. 3c), which is indicative of altered contractile regulation as observed with other HCM-causing sarcomeric mutations ${ }^{4-6}$. Therefore, the M305L actin mutation impairs Tpm-mediated inhibition of actomyosin associations and relaxation and promotes muscle force production.

Tpm and F-actin display a structurally synergistic relationship that depends on the complementarity of the surfaces between their three-dimensional forms ${ }^{19,23}$. Tpm's preshaped contour appears perfectly matched to the relatively flat face of actin subunits along the helical filament $19,21,23$. This structural matching permits a number of optimal interfacial contacts that are fundamental to the Gestalt-binding concept of Tpm regulatory behavior ${ }^{19,64}$. Roughly 30 favorable electrostatic contacts define weak, local F-actin-Tpm interactions ${ }^{24}$. These associations are likely to be essential for permitting the azimuthal, regulatory switching of Tpm, and electrostatic interaction maps reveal they establish an energy basin that biases Tpm to a location where actomyosin cycling is impeded ${ }^{24}$. For example, K326 and K328 of seven successive actin protomers attract oppositely charged residues along Tpm's entire length ${ }^{24}$. E334 was also among the actin residues purported to make weak, but stabilizing protein-protein contacts with $\mathrm{Tpm}^{24}$. These connections, in addition to Tn-Factin binding, were recently shown to be necessary for contractile inhibition 30,31 . 
In accordance with previous findings ${ }^{54}$, our MD simulations revealed that wildtype ACTC, in both the G- and F-actin forms, displays a high degree of flexibility (Fig. 5). Moreover, the wildtype monomer adopted several conformational substates that were separated by low energy barriers. The M305L mutation drastically reduced both actin's flexibility, especially that of SD2 and 4 as well as the stretch of residues that includes K326, K328, P333, and E334, and the population of conformers (Figs. 5 and 6). Additionally, using network analysis, we discovered that the M305L substitution changes the correlated motions of, and communication between, the protomer's subregions (Fig. 6). The surface loop containing P333 and E334 lost its ability to function independently, as found in $\mathrm{ACTC}^{\mathrm{WT}}$, which when combined with diminished flexibility could preclude the formation of certain, important actin conformations and substates required for Tpmmediated steric gating. Thus, optimal structural matching of the complementary F-actin-Tpm surface contours, and hence Gestalt-binding, may be impaired through disruption of the electrostatic interface, reducing the number of contacts. Weakening interfacial electrostatic associations would predictably deform the normal F-actin-Tpm energy landscape and promote regulatory imbalance. We therefore calculated the average electrostatic energy landscapes (Fig. 6d) from five individual maps, corresponding to the most populated conformational states adopted by wildtype and mutant ACTC during the CMD simulations. The electrostatic energy basin that normally biases Tpm to the inhibitory, $\mathrm{A} / \mathrm{B}$-state location along wildtype F-actin was shallower and narrower for the mutant.

Combining the in silico results, the diminished flexibility of P333 suggests an increased kinetic barrier that would slow Tpm $\mathrm{M}$-to-A/B state transitions while the shallower and less discretely localized electrostatic interaction energy basin implies a reduced thermodynamic driving force for the Tpm M-to-A/B state transition, thus enhancing contractile activation and prolonging inactivation. Overall, Tpm would be less confined azimuthally to an inhibitory location, which is consistent with our in vitro motility data that show significantly more mutant filaments are propelled by myosin relative to control (Table 4), and with the inability of mutant fly muscles to completely relax. Additionally, Cassell and Tobacman ${ }^{65}$ indicated that myosin-heads increase the affinity of Tpm for F-actin and Tpm enhances myosin binding to M-state actin, possibly through direct myosin-Tpm interactions ${ }^{66,67}$. Therefore, the M305L mutation could affect the S1 catalytic cycle through effects on F-actin-Tpm-myosin associations, and promote excessive force production and $\mathrm{HCM}^{68}$. However, these Tpm-myosin interactions were not tested in our in silico studies, nor do our experimental results, acquired from mutant IFMs, necessarily suggest they are impacted.

Our in vivo, in situ, and in vitro data, generated using numerous cardiac and skeletal muscle fly models, universally show that the M305L actin mutation results in aberrant thin filament-mediated contractile regulation. Mechanistically, our in silico findings imply that in addition to the complementary contours of Tpm and F-actin, a certain degree of actin subunit flexibility and intercommunity communication are required to facilitate the establishment and maintenance of the interfacial electrostatic contacts between the proteins. These contacts, in conjunction with the Tn complex, help establish the thin filament B-state and are vital for proper inhibition of muscle contraction. Therefore, by reducing actin protomer flexibility, the M305L substitution may disrupt F-actin-Tpm interaction and yield excessive force generation due to impaired relaxation, which are the earliest hallmarks of HCM in patients ${ }^{3}$ and the likely cause for myopathy in our animal models.

\section{Methods}

Construction of transgenes and transgenic Drosophila. UAS-Act57BGFP.WT and $-A c t 57 B^{W T}$ transgenes, the $A c t 88 F^{W T}$ genomic construct, and transgenic Drosophila were generated as described in Viswanathan et a ${ }^{30,31}$. UAS-Act57B GFP.M305L, -Act57B ${ }^{M 305 L}$, and $A c t 88 F^{M 305 L}$ transgenes were synthesized by site-directed mutagenesis using the QuikChange mutagenesis kit (Agilent Technologies) and custom primers.

Act57B ${ }^{\mathrm{M} 305 \mathrm{~L}}(+)$ primer - 5' GGTGGCACCACCCTGTACCCCGGTATT $3^{\prime}$

Act57B $\mathrm{B}^{\mathrm{M} 305 \mathrm{~L}}(-)$ primer $-5^{\prime}$ AATACCGGGGTACAGGGTGGTGCCACC $3^{\prime}$ Act88 $\mathrm{F}^{\mathrm{M} 305 \mathrm{~L}}(+)$ primer $-5^{\prime}$ GGCGGTACCACCCTGTACCCTGGTAAG $3^{\prime}$

Act88 $\mathrm{F}^{\mathrm{M} 305 \mathrm{~L}}(-)$ primer $-5^{\prime}$ CTTACCAGGGTACAGGGTGGTACCGCC $3^{\prime}$

Transgenic Drosophila were created using the PhiC31 integrase system, which ensures each gene integrates at an identical, predetermined genomic location and the results are directly comparable ${ }^{30,31,69}$. Therefore, phenotypic differences in control vs. mutant flies are immediately attributable to the single amino acid substitution in actin.

Drosophila stocks and husbandry. Tissue-specific transgene expression was achieved with the Gal4-UAS expression system ${ }^{70}$. Flies harboring a tissue-restricted Gal4 driver were crossed with flies containing constructs comprised of an UAS followed by an actin transgene $e^{30,31}$. The progeny inherit both genes and express the UAS-actin transgene exclusively in the desired muscle tissue. Hand $d^{4.2_{-} \text {Gal4 } \text { and }}$ UAS-Stinger flies were provided by Dr. Rolf Bodmer (Sanford Burnham Prebys Medical Discovery Institute, La Jolla, CA). 4XHand-Gal4 and Act88F-Gal4 were obtained from Drs. Zhe Han (George Washington University, Washington, D.C.), and Richard Cripps (San Diego State University, San Diego, CA) respectively. The TinC-Gal4 line was purchased from the Bloomington Stock Center. Act88F-null $\left(r y^{506}\right.$ KM88 $\left.e^{s}\right)$ Drosophila were obtained from Dr. John Sparrow (University of York, York, UK), and $M h c^{10}$ flies from Dr. Sanford Bernstein (San Diego State University, San Diego, CA $)^{71}$. All flies were raised on a standard cornmeal-yeastsucrose-agar medium at $25^{\circ} \mathrm{C}$ unless specified at $29^{\circ} \mathrm{C}$.

For all Gal4-UAS crosses, virgin females expressing the GAL4 protein, driven by a tissue-specific promoter, were mated with males carrying the UAS transgene. Act88F-Gal4/Act88F-Gal4; $M \mathrm{Cc}^{10} / \mathrm{Mhc}^{10}$ stocks were generated by standard mating procedures with balancer chromosomes and were crossed with UAS-Actin transgenic lines to generate flies expressing transgenic actin exclusively in the IFM, with a $50 \%$ reduction in IFM myosin.

Act88F transgenic Drosophila had endogenous wildtype $(+)$ actin genes on the third chromosomes, plus an additional $\left(A c t 88 F^{W T}\right.$ or Act88F $\left.F^{M 05 L}\right)$ transgene inserted into the second chromosomes (i.e. Act $88 F^{W T} / A c t 88 F^{W T} ;+/+$ and Act88F $\left.3305 L / A c t 88 F^{M 305 L} ;+/+\right)$. The transgenes were crossed into the Act $88 F$-null $r y^{506} \mathrm{KM} 88 e^{s}$ background by standard mating schemes using balancer chromosomes to generate Act88FWT/Act88FWT; $+/ K M 88$ and Act88FM305L/ Act88F $F^{M 305 L} ;+/ K M 88$ (designated as $A c t 88 F^{W T} / A c t 88 F^{W T}$; + and $A c t 88 F^{M 305 L /}$ Act $88 F^{M 305 L} ;+$, respectively). A second cross was performed to establish Act88F $F^{W T} / A c t 88 F^{W T} ; K M 88 / K M 88$ and Act88F $8305 L / A c t 88 F^{M 305 L} ; K M 88 / K M 88$ homozygous flies (called Act88FWT/Act88FWT and Act88F $F^{M 05 L} / A c t 88 F^{M 305 L}$, respectively). To generate heterozygotes, with one transgenic and one endogenous Act $88 F$ gene, $A c t 88 F^{W T} / A c t 88 F^{W T}$ or $A c t 88 F^{M 305 L} / A c t 88 F^{M 305 L}$ homozygotes were crossed with $w^{1118}$ yielding $A c t 88 F^{W T} ; K M 881+$ and $A c t 88 F^{M 305 L} ; K M 881+$ (referred to as $A c t 88 F^{W T} /+$ and $A c t 88 F^{M 305 L} /+$ ).

Confocal microscopy of the Drosophila heart. Virgin Hand-Gal4 female flies were crossed with males carrying the UAS-Act57B ${ }^{G F P . W T}$ or UAS-Act57BGFP.M305L transgene to assess the localization of ectopically expressed WT or M305L actin within the progeny's cardiac myofibrils. GFP-actin-expressing fly hearts were exposed, stained with TRITC-phalloidin, and imaged on a Leica TCS SPE RGBV confocal microscope at $\times 40$ and $\times 100$ magnification as previously detailed $31,72,73$

Cardiac physiological analysis. Three-week-old, female Hand-Gal4 $>, 4$ XHandGal4 $>$, or TinC-Gal4 > UAS-Act57B $B^{W T}$ or UAS-Act57B ${ }^{M 305 L}$ Drosophila hearts were exposed under artificial hemolymph $(\mathrm{AH})^{31,72}$. Cardiac performance was assessed as formerly outlined ${ }^{37,74}$. High-speed videos ( $\left.150 \mathrm{fps}\right)$ of semi-intact beating hearts were recorded with a Hamamatsu Orca-Flash 2.8 digital camera on a Leica DM5000B DIC microscope fitted with a $10 \times(0.30$ N.A. $)$ immersion lens. Cardiac performance was analyzed from the movies using Semi-automated Optical Heartbeat Analysis (SOHA) software ${ }^{36,37}$. M-modes, which provide an edge trace documenting heart wall movement over time, were generated via the program. Myogenic "cardiac output" was calculated as described in Blice-Baum et $\mathrm{al}^{74}$.

Measurement of cardiac dimensions post chemical treatment. Semi-intact hearts of three-week-old Hand-Gal4 > UAS-Act57B ${ }^{W T}$ and Hand-Gal4 > UAS$A c t 57 B^{M 305 L}$ females were imaged and filmed using a $20 \times(0.50$ N.A.) immersion lens ${ }^{40}$. The increase in heart tube diameters following incubation with EGTAEGTA, AM, to chelate extra- and intracellular $\mathrm{Ca}^{2+}$, and then blebbistatin, a small molecule myosin inhibitor, was measured and quantified as previously described 31,39 . 
Flight tests. Flight tests were performed on two-day-old male and female Drosophila as described in Drummond et al. ${ }^{75}$. Newly eclosed flies were aged for 2 days at $25^{\circ} \mathrm{C}$, or alternatively at $29^{\circ} \mathrm{C}$ where specified. Flies were released into the center of a plexiglass chamber with a light positioned at the top and each assigned a flight index based on direction of flight (6 for upward flight, 4 for horizontal, 2 for downward, or 0 for no flight). The average flight index was calculated by dividing the sum of the individual values by the number of animals tested for each line ${ }^{75}$.

IFM and myofibril imaging. IFM fluorescent microscopy and myofibril imaging of male and female Drosophila were performed as described previously $30,31,76,77$. Briefly, paraformaldehyde fixed, flash frozen thoraces of two-day-old flies were bisected after removing the heads and abdomens. Hemi-thoraces were stained with mouse anti- $\alpha$-actinin primary and donkey anti-mouse Alexa Fluor 488 secondary antibodies and TRITC-phalloidin, rinsed and imaged on an EVOS ${ }^{\oplus}$ FL cell imaging system (Life Technologies) at $\times 4$ magnification. IFM fibers were carefully cut, removed, and myofibrils gently teased apart and imaged on a Leica TCS SPE RGBV confocal microscope at $\times 100$ magnification. Measurement of thin filament lengths was performed using ImageJ software ${ }^{30,31,76,77}$.

IFM fiber mechanics. Skinned fibers were prepared from IFMs of 2-3 -day -old female flies as described previously ${ }^{46}$. Briefly, the fiber was mounted on the mechanics apparatus in relaxing solution (pCa 8.0, $12 \mathrm{mM} \mathrm{MgATP}, 30 \mathrm{mM}$ creatine phosphate, $600 \mathrm{U} \mathrm{mL}^{-1}$ creatine phosphokinase, $1 \mathrm{mM}$ free $\mathrm{Mg}^{2+}, 5 \mathrm{mM}$ EGTA, 20 mM N,N-Bis(2-hydroxyethyl)-2-aminoethanesulfonic acid (BES) (pH 7.0), $200 \mathrm{mM}$ ionic strength, adjusted with sodium methanesulfonate, $1 \mathrm{mM}$ dithiothreitol (DTT)) at $15^{\circ} \mathrm{C}$. The fiber was activated at various $\mathrm{Ca}^{2+}$ concentrations ( $\mathrm{pCa} 8.0$ to 4.0 ) by partial exchanges with activating solution (same as relaxing solution except pCa 4.0 )

Power, elastic modulus, and viscous modulus were measured using sinusoidal analysis as previously outlined ${ }^{46}$. A sinusoidal length change of $0.125 \%$ of the muscle length (ML) was applied to the fiber over a 0.5 to $600 \mathrm{~Hz}$ frequency range. Power $\left(\mathrm{W} \mathrm{m}^{-3}\right)$ was calculated as

$$
\pi f E_{\mathrm{v}}\left(\Delta L L^{-1}\right)^{2},
$$

where $f$ is the frequency of the length perturbation, $E_{v}$ is the viscous modulus at $f$, and $\Delta L L^{-1}$ is the amplitude of the length perturbation divided by the muscle length. The power-pCa data and elastic modulus-pCa data were fit using the following equation:

$$
\text { Power or Elastic modulus }=A\left(1+10^{\wedge}\left(\left(p C a_{50}-\mathrm{pCa}\right)(h)\right)\right)^{-1},
$$

where $A$ is the maximum power or elastic modulus, $\mathrm{pCa}_{50}$ is the inflection point at half maximal power or elastic modulus, and $h$ is the slope coefficient. Muscle apparent rate constants $2 \pi b$ and $2 \pi c$ were determined from plotting and fitting elastic modulus versus viscous modulus as previously described ${ }^{46,78}$.

IFM protein quantification. Quantitative western blots were performed on IFMs from Act88F-Gal4xyw and Act88F-Gal4 $>$ UAS-Act57B ${ }^{G F P . W T}$ Drosophila raised at $25^{\circ} \mathrm{C}$ and $29^{\circ} \mathrm{C}^{30}$. IFMs dissected from 8 flies were pooled as one biological replicate. Samples were immediately homogenized in Laemmli sample buffer, electrophoresed, and blotted onto a nitrocellulose membrane. Membranes were incubated overnight at $4{ }^{\circ} \mathrm{C}$ with primary rabbit anti-actin (Proteintech), rabbit anti-GFP (R\&D), and/or goat anti-GAPDH (Genscript) antibodies and were then probed with Donkey anti-rabbit and Donkey anti-goat IRDye secondary antibodies (LI-COR Biosciences) for 60-90 min at room temperature. The membranes were scanned using an Odyssey Infrared Imager $(\lambda=700$ and $800 \mathrm{~nm})$ and analyzed using Odyssey Application Software (v3.030, LI-COR Biosciences). Quantitation was performed on six biological samples with three technical replicates of each. Individual technical replicate values were averaged for each biological replicate. Mean values $( \pm$ SEM) of transgenic (GFP-labeled) and endogenous actin intensities were determined for the biological replicates and normalized to GAPDH intensities to verify changes in transgenic and endogenous actin abundance due to elevated temperature.

Actin purification from Drosophila IFM. Transgenic IFM actin from $A c t 88 F^{W T}$ / Act88F $F^{W T}$ and $A c t 88 F^{M 305 L} / A c t 88 F^{M 305 L}$ Drosophila was purified according to Razzaq, et al. ${ }^{43}$. IFMs from 30-40 flies were pulled and stored in York Modified Glycerol solution overnight at $-20^{\circ} \mathrm{C}$. High and low salt buffer extractions selectively isolated monomeric G-actin, which was then polymerized in high salt (to remove tropomyosin) and briefly pelleted at 436,000 $\mathrm{x} g$ in a Beckman TL-100 ultracentrifuge rotor. Pelleted actin was resolubilized in low salt buffer and mixed in a 1:1 molar ratio with Alexa568-phalloidin and left overnight at $4{ }^{\circ} \mathrm{C}$ to equilibrate. $10 \mathrm{nM}$ Alexa568-phalloidin-labeled F-actin was used for in vitro motility experiments.

In vitro motility. In vitro motility of Act $88 \mathrm{~F}^{\mathrm{WT}}$ and $\mathrm{Act} 88 \mathrm{~F}^{\mathrm{M} 305 \mathrm{~L}} \mathrm{~F}$-actin at $30^{\circ} \mathrm{C}$, in the presence or absence of $300 \mathrm{nM}$ porcine cardiac Tpm, was performed at rabbit psoas myosin concentrations between $12.5 \mu \mathrm{g} \mathrm{ml}^{-1}$ and $100 \mu \mathrm{g} \mathrm{ml}^{-1}$ in the following buffer: $25 \mathrm{mM} \mathrm{KCl}, 4 \mathrm{mM} \mathrm{MgCl}_{2}, 1 \mathrm{mM}$ EGTA, $25 \mathrm{mM}$ imidazole $\mathrm{pH}$
7.2, $10 \mathrm{mM}$ DTT, $1 \mathrm{mM}$ ATP, $2 \mathrm{mM}$ dextrose, 17 units $\mathrm{ml}^{-1}$ glucose oxidase, 125 units $\mathrm{ml}^{-1}$ catalase, and $0.5 \%$ methyl cellulose. Imaging was conducted on an Olympus IX73 microscope, and TRITC-phalloidin was excited using an X-CITE 120 LED lamp and a 531/40 filter. Emitted light was captured at 593/40 and detected on a Hamamatsu Flash 4LT EMCCD camera. Videos were recorded using HCI imaging software, converted to multipage TIF's, and imported into ImageJ. Tpm was pre-incubated with TRITC-phalloidin-labeled F-actin prior to flow cell addition. Duplicate assessments from two independent preparations of Act $88 \mathrm{~F}^{\mathrm{WT}}$ and Act88F ${ }^{\mathrm{M} 305 \mathrm{~L}}$ Drosophila IFM actin were carried out in parallel. Videos from 4-8 areas of the flow cell were recorded at 1-10 fps for 20 frames total, and filament velocities were measured via automated tracking using the ImageJ plugin, wrmtrack ${ }^{79}$. Identical filtering parameters were applied at each condition, i.e. myosin concentration and with or without Tpm, to limit program selection bias. In addition, a very sparse population of labeled F-actin $(\sim 1 \mathrm{nM})$ was used to reduce the number of filament collisions, which oftentimes confounds the tracking algorithm.

At each myosin concentration, and in the presence or absence of Tpm, Act $88 \mathrm{~F}^{\mathrm{WT}}$ and Act $88 \mathrm{~F}^{\mathrm{M} 305 \mathrm{~L}}$ average filament velocities were separately plotted as a function of standard deviation (determined from at least 200 filaments). Data were individually fit to a line $\left(R^{2}>\sim 0.7\right)$, and linear regression confirmed that the slopes were statistically equivalent. All data points were then combined and fit to a single line whose $y$-intercept was forced through the origin. The slope of the line represents, on average, the relationship between standard deviation and velocity, which was utilized to establish unique cutoff velocities at each condition tested. Filaments that displaced at an average velocity greater than cutoff were considered movers.

To determine the predicted standard deviation, S.D.pred, of a population of actin filaments with average velocity $V_{\text {avg }}$ measured via automated tracking, $V_{\text {avg }}$ from each condition was divided by the slope. The cutoff velocity $V_{\text {cut }}$ was then determined by the equation:

$$
V_{\text {cut }}=V_{\text {avg }}-0.5 \cdot \text { S.D. }{ }_{\text {pred }} \text {. }
$$

Thus, a filament was classified as a mover if its average velocity exceeded the average velocity of the population minus one-half of the population's predicted standard deviation. Percent filaments moving in each area of the flow cell was then determined by dividing the number of movers by filaments counted, and all technical and biological replicate values were averaged together.

Molecular modeling. Structural models of human $\alpha$-cardiac actin (ACTC) were generated with MODELLER ${ }^{80}$ using the bovine $\beta$-actin crystal structure (PDB ID 2BTF) as reference. Models of F-actin ACTC ${ }^{\mathrm{WT}}$ and $\mathrm{ACTC} \mathrm{C}^{\mathrm{M} 305 \mathrm{~L}}$ trimers were based on the near-atomic cryo-EM structure of $\alpha$-skeletal actin (PDB ID: 5JLF). The sequence identities between the ACTC target and those of the template structures are $94 \%$ (2BTF) and 99\% (5JLF). 20 structures were generated for G- and F-actin, and the MODELLER objective function along with the discrete optimized protein energy (DOPE) score were used for evaluation and selection of the final models. The RMSD values between the final G- and F-actin models and the template structures were $0.148 \AA$ and $0.194 \AA$, respectively. In all structural models, methylated histidine 73 was generated using the Schrödinger software suite ${ }^{81}$. ATP $\cdot \mathrm{Ca}^{2+}$ was placed in the active site of the G-actin models, while ADP $\cdot \mathrm{Mg}^{2+}$ was added to each protomer of the F-actin models by superposition with the experimental actin structures. Thus, the reference structures' nucleotide-protein interaction networks were preserved (Supplementary Fig. 12). For mutant $\mathrm{ACTC}^{\mathrm{M} 305 \mathrm{~L}}$, methionine 305 was replaced with leucine.

Classical and enhanced sampling MD simulations. A summary of the simulations is presented in Supplementary Table 3. A total of $4 \mu$ s simulations was performed with $2 \mu$ s enhanced sampling MD simulations. Unless otherwise stated, simulation trajectory analysis was performed using in-house python scripts and the MDAnalysis python package ${ }^{82,83}$, as well as VMD $1.9^{84}$. All simulations were conducted at the Computer Cluster of the North-German Supercomputing Alliance (HLRN).

Classical molecular dynamics (cMD) simulations were performed with NAMD $2.12^{85}$ and the CHARMM36 force field ${ }^{86,87}$. The CHARMM General Force Field ${ }^{88}$ was used to obtain parameters of methylated histidine. The structural models of $\mathrm{G}$ or F-actin ACTC ${ }^{\mathrm{WT}}$ and ACTC ${ }^{\mathrm{M} 305 \mathrm{~L}}$ were fully solvated with the TIP3P explicit water model ${ }^{89}$, and charges were neutralized by adding $\mathrm{Na}^{+}$counter ions. A minimum distance of $10 \AA$ between the solutes and the water box edges was used, leading to simulation systems with initial dimensions of $\sim 62 \times 88 \times 92 \AA^{3}$ for G actin and $147 \times 103 \times 108 \AA^{3}$ for F-actin. Periodic boundary conditions were applied for all simulations. Langevin dynamics and the Langevin piston method were used to maintain a constant temperature of $310 \mathrm{~K}$ and $1 \mathrm{~atm}$ pressure. The cutoff for van der Waals interactions and short-range electrostatics was set to $12 \AA$ and the particle-mesh Ewald method ${ }^{90}$ was used for long-range electrostatic interactions. The solvated G- or F-actin systems were initially energy-minimized and equilibrated. Subsequently, production run MD simulations were carried out for $200 \mathrm{~ns}$ with an integration time step of $2 \mathrm{fs}$. cMD simulations of G-actins were performed in triplicates. F-actin cMD simulations were performed in duplicates.

Accelerated molecular dynamics simulations (aMD) significantly enhance the sampling of the conformational space of proteins, with a few hundreds of nanoseconds aMD capturing events on the millisecond-timescale ${ }^{55}$. Therefore, by 
adding a boost potential to the dihedral angle energy of all individual atoms, in order to lower energy barriers of the protein system, which facilitates the transition between substates, aMD was used to enhance conformational sampling of the actin monomers ${ }^{56-58}$. Parameters for the threshold energy $E$ and the acceleration factor $\alpha$ were determined from the individual $200 \mathrm{~ns}$ cMD simulations of $\mathrm{ACTC}^{\mathrm{WT}}$ and $\mathrm{ACTC}^{\mathrm{M} 305 \mathrm{~L}}$, and were calculated as

$$
\begin{gathered}
E=\left\langle V_{\text {dihedral }}\right\rangle+3.5 \cdot N_{\text {residues }}, \\
\alpha=\frac{3.5}{5} \cdot N_{\text {residues }},
\end{gathered}
$$

where $N_{\text {residues }}$ equals the number of protein residues and $V_{\text {dihedral }}$ is the average dihedral energy determined from the cMD simulations. Two independent aMD simulations, for both wildtype and mutant M305L actin, were performed for $500 \mathrm{~ns}$ each, starting from the final structures of the $200 \mathrm{~ns}$ cMD simulations.

Principal component analysis. PCA of the individual $\mathrm{cMD}$ and aMD simulations was used for dimension reduction of the simulation data and to determine the dominant structural changes and correlated motions in the proteins by diagonalizing the covariance matrix, which was computed for the atomic positional fluctuations of all protein $\mathrm{C}_{\alpha}$ atoms. The eigenvectors with the largest eigenvaluesprincipal components 1 (PC1) and 2 (PC2) -reveal the dominant conformational changes and concerted atomic displacements in the proteins. To visualize the structural changes, $\mathrm{PC} 1$ and $\mathrm{PC} 2$ of the individual replicas were projected onto the respective average structures of the $\mathrm{cMD}$ or aMD simulations. In the case of $\mathrm{F}$-actin trimers, a single protomer (chain B) of ACTC ${ }^{\mathrm{WT}}$ or $\mathrm{ACTC}^{\mathrm{M} 305 \mathrm{~L}}$ of the individual replicas was subjected to PCA.

Computation of G-actin free energy landscapes. Two- and one-dimensional free energy profiles were calculated for $\mathrm{PC} 1$ and $\mathrm{PC} 2$ by reweighting the two combined aMD simulations of either $\mathrm{ACTC}^{\mathrm{WT}}$ or $\mathrm{ACTC}^{\mathrm{M} 305 \mathrm{~L}}$ using the PyReweighting toolkit ${ }^{59}$ and Maclaurin series expansion. A bin size of 5 was used for reweighting. PC1 and PC2 were determined through PCA of the first ACTC ${ }^{W T}$ aMD simulation, and all other wildtype and mutant aMDs were projected onto this subspace.

Dynamical network analysis and cross correlation. Dynamical network communities of highly correlated motions in wildtype and mutant actin monomers were constructed by computing the cross-correlation coefficient for the motion of all $\mathrm{C}_{\alpha}$ atom pairs ${ }^{60}$. Network connections (edges) were defined with a cutoff distance of $4.5 \AA$ and occupancy of more than $75 \%$ of the simulation time. The betweenness of an edge was calculated as the number of shortest paths that cross that edge. Network connections were weighted according to the cross-correlation data. The Girvan-Newman algorithm ${ }^{91}$ was used to define the network communities of correlated motions. Visualization of the dynamical network communities and the allosteric communication pathways was performed with the NetworkView plugin in $\mathrm{VMD}^{92}$.

Computation of F-actin-Tpm energy landscapes. F-actin-Tpm electrostatic energy landscapes were calculated as outlined previously $y^{21,22}$. Briefly, the actin monomer coordinates from the five predominant ACTC ${ }^{\mathrm{WT}}$ and $\mathrm{ACTC} \mathrm{C}^{\mathrm{M} 305 \mathrm{~L}}$ conformations, generated by hierarchical ensemble clustering ${ }^{93}$ of F-actin ACTC cMD simulations, were selected for landscape analysis. The 10 structures were first minimized in Charmm using the images function to include the contacts between monomers present in a filament, in the calculation. Through application of the filament helical symmetry operations, the resulting minimized structures were used to build filaments containing 16 actin monomers. Tpm, at a radius of $43 \AA$, was then azimuthally rotated in $2.5^{\circ}$ increments and longitudinally translated in $2.5 \AA$ increments to $\sim 300$ different grid points on the filament surfaces. The origins $(0,0)$ were set at the previously determined energy minimum of the inhibitory, $\mathrm{A} / \mathrm{B}$ configuration for wildtype F-actin- $\mathrm{Tpm}^{24}$, with positive longitude indicating the pointed end of Factin. Tpm at each grid point was then docked to these actin models in $0.5 \AA$ increments to a final radius of $39 \AA$. This docking method was necessary to resolve any poor Tpm-actin contacts that would occur with rotation and translation at the final radius, which are unresolvable through minimization. Conjugate gradient minimization was then performed to an energy gradient of $0.05 \mathrm{kcal} \mathrm{mol}^{-1} \AA^{-1}$. Electrostatic interaction energies between Tpm and the actin filaments were then calculated for each grid point and the results displayed in Gnuplot 5.2. The values of each grid point for the five wildtype and mutant F-actin-Tpm structures were then averaged and plotted. All plots were contoured with isolines between -1500 and $0 \mathrm{kcal} \mathrm{mol}^{-1}$ in increments of $300 \mathrm{kcal} \mathrm{mol}^{-1}$.

Statistical information. All statistical analyses were carried out using GraphPad Prism 7. When measured values were not normally distributed, data were logarithmically transformed and significance assessed via unpaired two-tailed $t$-tests, one-way ANOVAs with Tukey's multiple comparison tests or two-way ANOVAs with Bonferroni multiple comparison tests as indicated. Repeated measures ANOVAs with Tukey's multiple comparison tests were performed to study the effects of compounds (EGTA-EGTA,AM and blebbistatin) on the diameters of fly hearts. For flight data, Mann-Whitney tests were used, and for thin filament length measures, Kruskal-Wallis one-way ANOVAs with Dunn's post hoc tests were employed to assess significance. Large sample sizes, in these instances, assuaged concerns related to heterogeneity in variance. Pooled power: $\mathrm{Ca}^{2+}$ data were replotted, fit to the Hill equation, and significant differences in fit parameters $\left(\mathrm{pCa}_{50}\right.$ Hill coefficient, maximum power, $f_{\max }$, and cooperativity) determined via unpaired two-tailed Student's $t$ tests.

Reporting summary. Further information on research design is available in the Nature Research Reporting Summary linked to this article.

\section{Data availability}

Data supporting the findings of this manuscript are available from the corresponding authors upon reasonable request. A reporting summary for this Article is available as a Supplementary Information file. The source data underlying Figs. $2 \mathrm{~b}-\mathrm{i}, 3 \mathrm{~b}-\mathrm{c}, 4 \mathrm{a}, 5 \mathrm{c}$, $6 \mathrm{c}-\mathrm{d}$, Tables 1-4, supplementary figures 3A-C, 4, 5B-D, 6, 7A-B, E-F, 11 and supplementary tables 1 and 2 are provided in the source data file.

Received: 4 January 2019; Accepted: 30 March 2020; Published online: 15 May 2020

\section{References}

1. Maron, B. J., Maron, M. S. \& Semsarian, C. Genetics of hypertrophic cardiomyopathy after 20 years: clinical perspectives. J. Am. Coll. Cardiol. 60 , 705-715 (2012).

2. Semsarian, C., Ingles, J., Maron, M. S. \& Maron, B. J. New perspectives on the prevalence of hypertrophic cardiomyopathy. J. Am. Coll. Cardiol. 65, 1249-1254 (2015)

3. Green, E. M. et al. A small-molecule inhibitor of sarcomere contractility suppresses hypertrophic cardiomyopathy in mice. Science 351, 617-621 (2016).

4. Tardiff, J. C. Thin filament mutations: developing an integrative approach to a complex disorder. Circ. Res. 108, 765-782 (2011).

5. Marston, S. B. How do mutations in contractile proteins cause the primary familial cardiomyopathies? J. Cardiovasc Transl. Res. 4, 245-255 (2011).

6. Moore, J. R., Leinwand, L. \& Warshaw, D. M. Understanding cardiomyopathy phenotypes based on the functional impact of mutations in the myosin motor. Circ. Res. 111, 375-385 (2012).

7. Mogensen, J. et al. Clinical and genetic characteristics of alpha cardiac actin gene mutations in hypertrophic cardiomyopathy. J. Med. Genet. 41, e10 (2004).

8. Vang, S. et al. Actin mutations in hypertrophic and dilated cardiomyopathy cause inefficient protein folding and perturbed filament formation. FEBS $J$. 272, 2037-2049 (2005).

9. Mundia, M. M., Demers, R. W., Chow, M. L., Perieteanu, A. A. \& Dawson, J. F. Subdomain location of mutations in cardiac actin correlate with type of functional change. PLoS ONE 7, e36821 (2012).

10. Müller, M. et al. Functional characterization of the human alpha-cardiac actin mutations Y166C and M305L involved in hypertrophic cardiomyopathy. Cell Mol. Life Sci. 69, 3457-3479 (2012).

11. Dahari, M. \& Dawson, J. F. Do cardiac actin mutations lead to altered actomyosin interactions? Biochem. Cell Biol. 93, 330-334 (2015).

12. Gordon, A. M., Homsher, E. \& Regnier, M. Regulation of contraction in striated muscle. Physiol. Rev. 80, 853-924 (2000).

13. Lehman, W. Thin filament structure and the steric blocking model. Compr. Physiol. 6, 1043-1069 (2016).

14. Kiani, F. A., Lehman, W., Fischer, S. \& Rynkiewicz, M. J. Spontaneous transitions of actin-bound tropomyosin toward blocked and closed states. $J$. Gen. Physiol. 151, 4-8 (2019).

15. Brown, J. H. \& Cohen, C. Regulation of muscle contraction by tropomyosin and troponin: how structure illuminates function. Adv. Protein Chem. 71, 121-159 (2005).

16. Hitchcock-DeGregori, S. E. Tropomyosin: function follows structure. $A d v$. Exp. Med Biol. 644, 60-72 (2008).

17. Hitchcock-DeGregori, S.E. \& Barua, B. in Fibrous Proteins: Structures and mechanisms (Springer, 2017). https://doi.org/10.1007/978-3-319-49674-0_9.

18. Heald, R. W. \& Hitchcock-DeGregori, S. E. The structure of the amino terminus of tropomyosin is critical for binding to actin in the absence and presence of troponin. J. Biol. Chem. 263, 5254-5259 (1988).

19. Holmes, K. C. \& Lehman, W. Gestalt-binding of tropomyosin to actin filaments. J. Muscle Res. Cell Motil. 29, 213-219 (2008).

20. Tobacman, L. S. Cooperative binding of tropomyosin to actin. Adv. Exp. Med. Biol. 644, 85-94 (2008)

21. Lehman, W., Orzechowski, M., Li, X. E., Fischer, S. \& Raunser, S. Gestaltbinding of tropomyosin on actin during thin filament activation. J. Muscle Res. Cell Motil. 34, 155-163 (2013). 
22. Rynkiewicz, M. J. et al. Tropomyosin must interact weakly with actin to effectively regulate thin filament function. Biophys. J. 113, 2444-2451 (2017).

23. Li, X. E., Holmes, K. C., Lehman, W., Jung, H. \& Fischer, S. The Shape and flexibility of tropomyosin coiled coils: implications for actin filament assembly and regulation. J. Mol. Biol. 395, 327-339 (2010).

24. Li, X. E. et al. Tropomyosin position on F-actin revealed by EM reconstruction and computational chemistry. Biophys. J. 100, 1005-1013 (2011).

25. Brown, J. H. et al. Structure of the mid-region of tropomyosin: bending and binding sites for actin. Proc. Natl Acad. Sci. USA 102, 18878-18883 (2005).

26. Barua, B., Pamula, M. C. \& Hitchcock-DeGregori, S. E. Evolutionarily conserved surface residues constitute actin binding sites of tropomyosin. Proc. Natl Acad. Sci. USA 108, 10150-10155 (2011).

27. Barua, B. Periodicities designed in the tropomyosin sequence and structure define its functions. Bioarchitecture 3, 51-56 (2013).

28. von der Ecken, J. et al. Structure of the F-actin-tropomyosin complex. Nature 519, 114-117 (2015).

29. Marston, S. et al. Mutations in repeating structural motifs of tropomyosin cause gain of function in skeletal muscle myopathy patients. Hum. Mol. Genet. 22, 4978-4987 (2013).

30. Viswanathan, M. C., Blice-Baum, A. C., Schmidt, W., Foster, D. B. \& Cammarato, A. Pseudo-acetylation of K326 and K328 of actin disrupts Drosophila melanogaster indirect flight muscle structure and performance. Front. Physiol. 6, 116 (2015).

31. Viswanathan, M. C. et al. Distortion of the actin A-triad results in contractile disinhibition and cardiomyopathy. Cell Rep. 20, 2612-2625 (2017).

32. Cammarato, A. et al. A mighty small heart: the cardiac proteome of adult Drosophila melanogaster. PLoS ONE 6, el8497 (2011).

33. Shah, A. P. et al. Cardiac remodeling in Drosophila arises from changes in actin gene expression and from a contribution of lymph gland-like cells to the heart musculature. Mech. Dev. 128, 222-233 (2011).

34. Hiromi, Y. \& Hotta, Y. Actin gene mutations in Drosophila; heat shock activation in the indirect flight muscles. EMBO J. 4, 1681-1687 (1985).

35. Miller, A. in Biology of Drosophila (ed Demerec, M) (Wiley, 1950).

36. Fink, M. et al. A new method for detection and quantification of heartbeat parameters in Drosophila, zebrafish, and embryonic mouse hearts. Biotechniques 46, 101-113 (2009).

37. Cammarato, A., Ocorr, S. \& Ocorr, K. Enhanced assessment of contractile dynamics in Drosophila hearts. Biotechniques 58, 77-80 (2015).

38. Duffy, J. B. GAL4 system in Drosophila: a fly geneticist's Swiss army knife. Genesis 34, 1-15 (2002).

39. Kronert, W. A. et al. Prolonged cross-bridge binding triggers muscle dysfunction in a Drosophila model of myosin-based hypertrophic cardiomyopathy. Elife 7, https://doi.org/10.7554/eLife.38064 (2018).

40. Viswanathan, M. C., Kaushik, G., Engler, A. J., Lehman, W. \& Cammarato, A. A Drosophila melanogaster model of diastolic dysfunction and cardiomyopathy based on impaired troponin-T function. Circ. Res. 114, e6-e17 (2014).

41. Fedorov, V. V. et al. Application of blebbistatin as an excitation-contraction uncoupler for electrophysiologic study of rat and rabbit hearts. Heart Rhythm 4, 619-626 (2007).

42. Beall, C. J. \& Fyrberg, E. Muscle abnormalities in Drosophila melanogaster heldup mutants are caused by missing or aberrant troponin-I isoforms. J. Cell Biol. 114, 941-951 (1991).

43. Razzaq, A. et al. Actin residue glu(93) is identified as an amino acid affecting myosin binding. J. Biol. Chem. 274, 28321-28328 (1999).

44. Cammarato, A. et al. Drosophila muscle regulation characterized by electron microscopy and three-dimensional reconstruction of thin filament mutants. Biophys. J. 86, 1618-1624 (2004).

45. Cammarato, A. et al. Myosin transducer mutations differentially affect motor function, myofibril structure, and the performance of skeletal and cardiac muscles. Mol. Biol. Cell 19, 553-562 (2008).

46. Swank, D. M. Mechanical analysis of Drosophila indirect flight and jump muscles. Methods 56, 69-77 (2012).

47. Winter JMd et al. Mutation-specific effects on thin filament length in thin filament myopathy. Ann. Neurol. 79, 959-969 (2016).

48. Röper, K., Mao, Y. \& Brown, N. H. Contribution of sequence variation in Drosophila actins to their incorporation into actin-based structures in vivo. J. Cell Sci. 118, 3937-3948 (2005).

49. Gerson, J. H., Bobkova, E., Homsher, E. \& Reisler, E. Role of Residues 311/312 in Actin-Tropomyosin Interaction In vitro motility study using yeast actin mutant E311A/R312A. J. Biol. Chem. 274, 17545-17550 (1999).

50. VanBuren, P., Palmiter, K. A. \& Warshaw, D. M. Tropomyosin directly modulates actomyosin mechanical performance at the level of a single actin filament. Proc. Natl Acad. Sci. 96, 12488-12493 (1999).

51. Strand, J., Nili, M., Homsher, E., Tobacman, L. S. Modulation of myosin function by isoform-specific properties of S. cerevisiae and muscle tropomyosins. J. Biol. Chem. 276, 34832-9 (2001).
52. Barua, B., Winkelmann, D. A., White, H. D. \& Hitchcock-DeGregori, S. E. Regulation of actin-myosin interaction by conserved periodic sites of tropomyosin. Proc. Natl Acad. Sci. USA 109, 18425-18430 (2012).

53. Amadei, A., Linssen, A. B. \& Berendsen, H. J. Essential dynamics of proteins. Proteins: Struct. Funct. Bioinform. 17, 412-425 (1993).

54. Splettstoesser, T., Holmes, K. C., Noe, F. \& Smith, J. C. Structural modeling and molecular dynamics simulation of the actin filament. Proteins 79, 2033-2043 (2011).

55. Pierce, L. C. et al. Routine access to millisecond time scale events with accelerated molecular dynamics. J. Chem. Theory Comput. 8, 2997-3002 (2012).

56. Hamelberg, D., Mongan, J. \& McCammon, J. A. Accelerated molecular dynamics: a promising and efficient simulation method for biomolecules. J. Chem. Phys. 120, 11919-11929 (2004).

57. Bucher, D., Grant, B. J., Markwick, P. R. \& McCammon, J. A. Accessing a hidden conformation of the maltose binding protein using accelerated molecular dynamics. PLoS Comput. Biol. 7, e1002034 (2011).

58. Markwick, P. R. \& McCammon, J. A. Studying functional dynamics in biomolecules using accelerated molecular dynamics. Phys. Chem. Chem. Phys. 13, 20053-20065 (2011).

59. Miao, Y. et al. Improved reweighting of accelerated molecular dynamics simulations for free energy calculation. J. Chem. Theory Comput. 10, 2677-2689 (2014).

60. Sethi, A., Eargle, J., Black, A. A. \& Luthey-Schulten, Z. Dynamical networks in tRNA: protein complexes. Proc. Natl Acad. Sci. 106, 6620-6625 (2009).

61. Xie, H. B. et al. The NADPH metabolic network regulates human $a B-$ crystallin cardiomyopathy and reductive stress in Drosophila melanogaster. PLoS Genet 9, e1003544, https://doi.org/10.1371/journal.pgen.1003544 (2013).

62. Yu, L., Daniels, J., Glaser, A. E. \& Wolf, M. J. Raf-mediated cardiac hypertrophy in adult Drosophila. Dis. Model Mech. 6, 964-976 (2013).

63. Brutsaert, D. L. \& Sys, S. U. Relaxation and diastole of the heart. Physiol. Rev. 69, 1228-1315 (1989).

64. Wegner, A. The interaction of alpha, alpha- and alpha, beta-tropomyosin with actin filaments. FEBS Lett. 119, 245-248 (1980).

65. Cassell, M. \& Tobacman, L. S. Opposite effects of myosin subfragment 1 on binding of cardiac troponin and tropomyosin to the thin filament. J. Biol. Chem. 271, 12867-12872 (1996).

66. Lehrer, S. S. The regulatory switch of the muscle thin filament: $\mathrm{Ca} 2+$ or myosin heads? J. Muscle Res. Cell Motil. 15, 232-236 (1994).

67. Lehrer, S. S. The 3-state model of muscle regulation revisited: is a fourth state involved? J. Muscle Res. Cell Motil. 32, 203 (2011).

68. Vera, C. D. et al. Myosin motor domains carrying mutations implicated in early or late onset hypertrophic cardiomyopathy have similar properties. $J$. Biol. Chem. 294, 17451-17462 (2019).

69. Groth, A. C., Fish, M., Nusse, R. \& Calos, M. P. Construction of transgenic Drosophila by using the site-specific integrase from phage phiC31. Genetics 166, 1775-1782 (2004).

70. Brand, A. H. \& Perrimon, N. Targeted gene expression as a means of altering cell fates and generating dominant phenotypes. Development 118, 401-415 (1993).

71. O’Donnell, P. T., Collier, V. L., Mogami, K. \& Bernstein, S. I. Ultrastructural and molecular analyses of homozygous-viable Drosophila melanogaster muscle mutants indicate there is a complex pattern of myosin heavy-chain isoform distribution. Genes Dev. 3, 1233-1246 (1989).

72. Vogler, G., Ocorr, K. Visualizing the beating heart in Drosophila. J. Vis. Exp. https://doi.org/10.3791/1425 (2009).

73. Alayari, N. N. et al. Fluorescent labeling of Drosophila heart structures. J. Vis. Exp. https://doi.org/10.3791/1423 (2009).

74. Blice-Baum, A. C. et al. Modest overexpression of FOXO maintains cardiac proteostasis and ameliorates age-associated functional decline. Aging Cell 16, 93-103 (2017).

75. Drummond, D. R., Hennessey, E. S. \& Sparrow, J. C. Characterisation of missense mutations in the Act $88 \mathrm{~F}$ gene of Drosophila melanogaster. Mol. Gen. Genet. 226, 70-80 (1991).

76. Haigh, S. E. et al. Drosophila indirect flight muscle specific Act88F actin mutants as a model system for studying congenital myopathies of the human ACTA1 skeletal muscle actin gene. Neuromuscul. Disord. 20, 363-374 (2010).

77. Cammarato, A. et al. Structural basis for myopathic defects engendered by alterations in the Myosin rod. J. Mol. Biol. 414, 477-484 (2011).

78. Kawai, M. \& Brandt, P. W. Sinusoidal analysis: a high resolution method for correlating biochemical reactions with physiological processes in activated skeletal muscles of rabbit, frog and crayfish. J. Muscle Res Cell Motil. 1, 279-303 (1980).

79. Nussbaum-Krammer, C. I., Neto, M. F., Brielmann, R. M., Pedersen, J. S., Morimoto, R. I. Investigating the spreading and toxicity of prion-like proteins using the metazoan model organism C. elegans. J. Vis. Exp. 52321. https://doi. org/10.3791/52321 (2015). 
80. Sali, A. \& Blundell, T. L. Comparative protein modelling by satisfaction of spatial restraints. J. Mol. Biol. 234, 779-815 (1993).

81. Release. Schrödinger Suite 2016-2 Protein Preparation Wizard; Epik; Impact; Prime; MacroModel, Schrödinger, LLC, New York, NY, 2016.) (2016-2).

82. Michaud-Agrawal, N., Denning, E. J., Woolf, T. B. \& Beckstein, O. MDAnalysis: a toolkit for the analysis of molecular dynamics simulations. $J$. Computat. Chem. 32, 2319-2327 (2011).

83. Gowers, R.J. et al. MDAnalysis: a Python Package for the Rapid Analysis of Molecular Dynamics Simulations (Los Alamos National Lab. (LANL), Los Alamos, NM, 2019).

84. Humphrey, W., Dalke, A. \& Schulten, K. VMD: visual molecular dynamics. J. Mol. Graph 14, 27-38 (1996).

85. Phillips, J. C. et al. Scalable molecular dynamics with NAMD. J. Comput. Chem. 26, 1781-1802 (2005).

86. Best, R. B. et al. Optimization of the additive CHARMM all-atom protein force field targeting improved sampling of the backbone $\phi, \psi$ and side-chain $\chi 1$ and $\chi^{2}$ dihedral angles. J. Chem. Theory Comput. 8, 3257-3273 (2012).

87. Huang, J. et al. CHARMM $36 \mathrm{~m}$ : an improved force field for folded and intrinsically disordered proteins. Nat. Methods 14, 71-73 (2017).

88. Vanommeslaeghe, K. \& MacKerell, A. D. Jr. Automation of the CHARMM General Force Field (CGenFF) I: bond perception and atom typing. J. Chem. Inf. Model 52, 3144-3154 (2012)

89. Jorgensen, W. L., Chandrasekhar, J., Madura, J. D., Impey, R. W. \& Klein, M. L. Comparison of simple potential functions for simulating liquid water. J. Chem. Phys. 79, 926-935 (1983).

90. Darden, T., York, D. \& Pedersen, L. Particle mesh Ewald: An N. $\log (\mathrm{N})$ method for Ewald sums in large systems. J. Chem. Phys. 98, 10089-10092 (1993).

91. Girvan, M. \& Newman, M. E. Community structure in social and biological networks. Proc. Natl Acad. Sci. 99, 7821-7826 (2002).

92. Eargle, J. \& Luthey-Schulten, Z. NetworkView: 3D display and analysis of protein. RNA Interact. Netw. Bioinform. 28, 3000-3001 (2012).

93. Kelley, L. A., Gardner, S. P. \& Sutcliffe, M. J. An automated approach for clustering an ensemble of NMR-derived protein structures into conformationally related subfamilies. Protein Eng. Des. Selection 9, 1063-1065 (1996).

\section{Acknowledgements}

The authors thank the North-German Supercomputing Alliance (HLRN) for providing HPC resources that contributed to the results reported in this paper. The work was funded by Deutsche Forschungsgemeinschaft (DFG) grant PR 1478/2-1 (M.P.) and by NIH R01HL124091 (A.C.), T32HL007227 (W.S.), R37HL036153, and R01HL036153 (W.L.), and AHA 17POST33630159 (W.S.).

\section{Author contributions}

M.C.V., W.S., P.F., M.J.R., A.M., W.L., D.M.S., M.P., and A.C. conceived and designed the experiments. M.C.V., W.S., P.F., C.S.N., M.J.R., A.M., and M.P.acquired the data M.C.V., W.S., P.F., C.S.N., M.J.R., A.M., W.L., D.M.S., M.P., and A.C. analyzed and interpreted the data. M.C.V., W.S., P.F., C.S.N., M.J.R., W.L., D.M.S., M.P., and A.C. drafted and revised the manuscript.

\section{Competing interests}

The authors declare no competing interests.

\section{Additional information}

Supplementary information is available for this paper at https://doi.org/10.1038/s41467020-15922-5.

Correspondence and requests for materials should be addressed to M.P. or A.C.

Peer review information Nature Communications thanks Michael Geeves and the other, anonymous, reviewer(s) for their contribution to the peer review of this work.

Reprints and permission information is available at http://www.nature.com/reprints

Publisher's note Springer Nature remains neutral with regard to jurisdictional claims in published maps and institutional affiliations.

(c) Open Access This article is licensed under a Creative Commons Attribution 4.0 International License, which permits use, sharing, adaptation, distribution and reproduction in any medium or format, as long as you give appropriate credit to the original author(s) and the source, provide a link to the Creative Commons license, and indicate if changes were made. The images or other third party material in this article are included in the article's Creative Commons license, unless indicated otherwise in a credit line to the material. If material is not included in the article's Creative Commons license and your intended use is not permitted by statutory regulation or exceeds the permitted use, you will need to obtain permission directly from the copyright holder. To view a copy of this license, visit http://creativecommons.org/ licenses/by/4.0\%

(C) The Author(s) 2020 